\title{
Analysis and Efficient Evaluation of the BER of OSTBCs With Imperfect Channel Estimation in Arbitrarily Correlated Fading Channels
}

\author{
Lennert Jacobs, Student Member, IEEE, George C. Alexandropoulos, Member, IEEE, \\ Marc Moeneclaey, Fellow, IEEE, Herwig Bruneel, and P. Takis Mathiopoulos, Senior Member, IEEE
}

\begin{abstract}
An analysis of the exact bit error rate (BER) performance of orthogonal space-time block codes (OSTBCs) with maximum-likelihood detection in the presence of channel estimation errors is presented. The possibly correlated coefficients of the multiple-input multiple-output (MIMO) propagation channel are assumed to be affected by flat block fading and the transmitted symbols belong to a pulse amplitude modulation or quadrature amplitude modulation signal constellation. For both square and nonsquare OSTBCs, we derive approximate and exact BER expressions, irrespective of the distribution of the fading. It is also shown how the exact expressions can be efficiently and accurately evaluated using numerical integration techniques. Their application to the arbitrarily correlated Nakagami- $m$ fading channel is presented and an efficient importance sampling technique is derived. As the high diversity order resulting from the application of OSTBCs gives rise to small BER values, the numerical evaluation of the presented BER expressions is much faster than straightforward Monte Carlo simulations. BER results have shown the impact of both imperfect channel estimation and antenna correlation on the performance of MIMO OSTBC systems. It is also shown that under highly correlated conditions, antenna correlation is the major source of BER degradation.
\end{abstract}

Index Terms-Channel estimation, correlated MIMO channels, error analysis, Nakagami fading, OSTBC.

\section{INTRODUCTION}

I $\mathrm{N}$ wireless communications, the detrimental effect of channel fading on the system performance can be tackled by using a proper diversity scheme, which provides the receiver

Manuscript received March 09, 2010; revised August 20, 2010 and December 17, 2010; accepted January 24, 2011. Date of publication February 14, 2011; date of current version May 18, 2011. The associate editor coordinating the review of this manuscript and approving it for publication was Prof. Ali Ghrayeb. This work was supported by the European Commission in the framework of the FP7 Network of Excellence in Wireless COMmunications NEWCOM++ (Contract 216715). L. Jacobs also gratefully acknowledges the support from the Fund for Scientific Research in Flanders (FWO-Vlaanderen). The material in this paper was presented in part at the IEEE Symposium on Personal, Indoor, and Mobile Radio Communications, Cannes, France, 2008 and in Personal, Indoor, and Mobile Radio Communications (PIMRC), Tokyo, Japan, 2009.

L. Jacobs, M. Moeneclaey, and H. Bruneel are with the Department of Telecommunications and Information Processing, Ghent University, B-9000 Gent, Belgium (e-mail: Lennert.Jacobs@telin.ugent.be; Marc.Moeneclaey@telin.ugent.be; Herwig.Bruneel@telin.ugent.be).

G. C. Alexandropoulos is with the Computer Engineering and Informatics Department, School of Engineering, University of Patras, GR-26500 Rio-Patras, Greece, and also with the Institute of Informatics and Telecommunications, National Centre for Scientific Research-“Demokritos", GR-15310 Athens, Greece (e-mail: alexandg@ieee.org).

P. T. Mathiopoulos is with the National Observatory of Athens, Institute for Space Applications and Remote Sensing, Palea Penteli, GR-15236 Athens, Greece (e-mail: mathio@ieee.org).

Digital Object Identifier 10.1109/TSP.2011.2114654 with multiple copies of the same signal through different paths or branches which are independent in time, frequency or space. Spatial diversity can be achieved by combining multiple-input mutiple-output (MIMO) systems with space-time coding. In this paper, we focus on the appealing transmit diversity technique of orthogonal space-time block coding, which achieves full diversity gain and results in a very simple maximum-likelihood (ML) detection algorithm, based only on linear processing at the receiver [1]-[3]. Owing to these beneficial properties, the error performance of orthogonal space-time block codes (OSTBCs) has been examined to great extent in various fading environments under the assumption that perfect channel state information (PCSI) is available at the receiver [4]-[11].

In practical wireless scenarios, however, the assumption of PCSI is not valid since the receiver has to estimate the channel. Typically, the channel is estimated with the aid of known pilot symbols sent among the data [12], although blind or semi-blind joint channel estimation and detection techniques can also be applied [13], [14]. In the past, the performance of OSTBC systems in the presence of imperfect channel state information (ICSI) has been studied considering Rayleigh fading [15]-[20]. For example, under the assumption of independent and identically distributed (i.i.d.) fading, an exact closed-form BER expression for square OSTBCs was derived in [15] for pilot-based minimum mean-square error (MMSE) channel estimation and pulse amplitude modulation (PAM) or quadrature amplitude modulation (QAM) constellations. In [16], this analysis was extended to correlated fading channels. In the case of $M$-ary phase-shift keying ( $M$-PSK) constellations, exact closed-form BER expressions as well as tight upper bounds were given in [17] for both decision feedback (DF) and pilot-based MMSE channel estimation. Asymptotically tight pairwise error probability (PEP) expressions were obtained in [18] for quite general STBCs with coherent and noncoherent receivers using an eigenvalue approach. Using the characteristic function of the decision variable, an exact closed-form expression for the PEP of both orthogonal and nonorthogonal space-time codes with least-squares (LS) channel estimation was derived in [19]. In [20], this result was extended to the case of correlated Rayleigh fading with receive correlation only. For arbitrarily distributed fading channels and $M$-PSK constellations, the symbol error probability (SEP) as well as the decoding error probability (DEP) of square OSTBCs have been examined in [21]. However, in [22], we have shown that the method in [21] to compute the error performance includes an approximation and cannot easily be generalized to QAM and PAM constellations. 
The contribution of this paper is twofold. First, we provide an exact analytical BER analysis for square and nonsquare OSTBCs with $M$-ary PAM or QAM constellations and ICSI, under flat-fading channels with an arbitrary joint probability density function (PDF); in spite of the numerous published papers dealing with the impact of ICSI on the performance of OSTBCs, e.g., [15], [17]-[21], and [23], such exact analysis is still lacking in the open literature. Second, we provide a simple approximate BER expression based on treating the symbol interference due to imperfect channel estimation as white Gaussian noise. Although this expression is in general not asymptotically exact, it yields quite accurate BER results in many practical applications.

The remainder of this paper is organized as follows. In Section II, a mathematical model for an OSTBC MIMO system is introduced. Section III presents the well-known pilot-based LS channel estimation algorithm and considers a mismatched ML receiver that uses the estimated channel as if it was the true channel. In Section IV, we present the exact BER expressions for OSTBCs under generalized fading conditions with ICSI, whereas Section V provides a simple approximate BER expression. Section VI deals with the efficient and accurate evaluation of the exact BER expressions, and outlines a method for Monte Carlo integration with importance sampling. Section VII concentrates on the evaluation of the BER in the particular case of arbitrarily correlated Nakagami- $m$ fading channels. In Section VIII, Monte Carlo simulations of the receiver operations confirm our efficient numerical evaluation methods and the impact of several system and channel parameters on the BER performance is investigated. Moreover, the accuracy of the approximate BER expressions is discussed. Finally, conclusions are drawn in Section IX.

Throughout this paper, $\otimes$ denotes the Kronecker product, $j^{2}=-1$ and the superscripts $*, T$, and $H$ represent complex conjugate, transpose, and conjugate transpose, respectively. $\mathbb{E}[x]$ and $|x|$ denote the expected value and the absolute value of $x$, respectively, whereas $\operatorname{diag}\left\{x_{1}, x_{2}, \ldots, x_{N}\right\}$ stands for an $N \times N$ diagonal matrix whose diagonal elements starting from the upper left corner are $x_{1}, x_{2}, \ldots, x_{N}$. Vectors and matrices are denoted by boldface lowercase letters and boldface capital letters, respectively. The norm of $\mathbf{x}$ is denoted by $\|\mathbf{x}\|$, whereas $\|\mathbf{X}\|_{F}, \operatorname{tr}(\mathbf{X})$, and $\sqrt{\mathbf{X}}$ refer to the Frobenius norm, the trace, and the element-wise square root of $\mathbf{X}$, respectively. The column vector that is obtained by stacking the columns of the matrix $\mathbf{X}$ is denoted by $\operatorname{vec}(\mathbf{X})$.

\section{SYSTEM MODEL}

Let us consider a MIMO wireless communication system with $L_{\mathrm{t}}$ transmit and $L_{\mathrm{r}}$ receive antennas. The transmitted data symbols at each transmit antenna are assumed to be coded according to a complex OSTBC [2], [3], which is defined by an $L_{\mathrm{t}} \times K_{\mathrm{C}}$ coded symbol matrix $\mathbf{C}$, where $K_{\mathrm{c}}$ denotes the block length. The entries of $\mathrm{C}$ are linear combinations of $N_{\mathrm{S}}$ information symbols $s_{i}=s_{i, \mathrm{R}}+j s_{i, \mathrm{I}}, 1 \leq i \leq N_{\mathrm{s}}$, with $s_{i, \mathrm{R}}$ and $s_{i, \mathrm{I}}$ denoting the real and imaginary parts of $s_{i}$, respectively, and their complex conjugate $s_{i}^{*}$, such that $\mathbf{C}$ can be written as

$$
\mathbf{C}=\sum_{i=1}^{N_{\mathrm{s}}}\left(\mathbf{C}_{i} s_{i}+\mathbf{C}_{i}^{\prime} s_{i}^{*}\right)
$$

where the $L_{\mathrm{t}} \times K_{\mathrm{c}}$ matrices $\mathbf{C}_{i}$ and $\mathbf{C}_{i}^{\prime}$ comprise the constant complex coefficients of the information symbols $s_{i}$ and $s_{i}^{*}$, respectively, in the matrix C. Since scaling of the code matrix $\mathbf{C}$ does not affect its orthogonality, we scale $\mathbf{C}$ in such way that it satisfies the following orthogonality condition

$$
\mathbf{C C}^{H}=\lambda\|\mathbf{s}\|^{2} \mathbf{I}_{L_{\mathrm{t}}}
$$

where $\lambda \triangleq K_{\mathrm{c}} / N_{\mathrm{s}}, \mathbf{s}=\left(s_{1}, s_{2}, \ldots, s_{N_{\mathrm{s}}}\right)^{T}$ is the data symbol vector, and $\mathbf{I}_{L_{\mathrm{t}}}$ denotes the $L_{\mathrm{t}} \times L_{\mathrm{t}}$ identity matrix. We consider a normalized information symbol constellation $\Psi$, such that $\mathbb{E}\left[\left|s_{i}\right|^{2}\right]=1$, and (2) yields

$$
\frac{1}{L_{\mathrm{t}} K_{\mathrm{C}}} \mathbb{E}\left[\|\mathbf{C}\|_{\mathrm{F}}^{2}\right]=1 \text {. }
$$

Also, from (1) and (2), it follows that

$$
\begin{aligned}
\mathbf{C}_{i} \mathbf{C}_{n}^{H}+\mathbf{C}_{n}^{\prime}{\mathbf{C}_{i}^{\prime}}^{H} & =\lambda \delta_{i-n} \mathbf{I}_{L_{\mathrm{t}}} \\
\mathbf{C}_{i} \mathbf{C}_{n}^{\prime H}+\mathbf{C}_{n} \mathbf{C}_{i}^{\prime H} & =\mathbf{0}_{L_{\mathrm{t}}}
\end{aligned}
$$

where $1 \leq i, n \leq N_{\mathrm{s}}$, and $\delta_{i-n}$ and $\mathbf{0}_{L_{\mathrm{t}}}$ denote the Kronecker delta and an $L_{\mathrm{t}} \times L_{\mathrm{t}}$ all-zero matrix, respectively. For square OSTBCs, i.e., $L_{\mathrm{t}}=K_{\mathrm{c}}$, it is readily verified that

$$
\mathbf{C}^{H} \mathbf{C}=\mathbf{C C}^{H} \text {. }
$$

Examples of square OSTBCs are the $2 \times 2$ Alamouti code [1] and the $4 \times 4$ codes given in [2, eq. (40)], [3, eq. (62)], and [24, eq. (41)]. Uncoded single-input multiple-output (SIMO) systems can be treated as a special case of square OSTBCs, with $L_{\mathrm{t}}=N_{\mathrm{s}}=1$, and coefficient matrices $\mathbf{C}_{1}=1$ and $\mathbf{C}_{1}^{\prime}=0$.

Data transmission is organized in frames consisting of $K_{\mathrm{p}}$ known pilot symbols used for channel estimation and $K$ coded data symbols per transmit antenna, with $K$ being a multiple of $K_{\mathrm{c}}$. In this way, $K / K_{\mathrm{c}}$ coded symbol matrices $\mathbf{C}(k)$, with $k$ denoting the block index, are sent within one frame. Also, we assume that the $L_{\mathrm{t}} \times K_{\mathrm{p}}$ pilot matrix $\mathbf{C}_{\mathrm{p}}$ satisfies

$$
\frac{1}{L_{\mathrm{t}} K_{\mathrm{p}}} \mathbb{E}\left[\left\|\mathbf{C}_{\mathrm{p}}\right\|_{\mathrm{F}}^{2}\right]=1 \text {. }
$$

The propagation channels between each transmit and receive antenna pair are affected by flat fading with an arbitrary distribution and additive white Gaussian noise (AWGN) with power spectral density $N_{0}$; in addition, we allow correlation between the propagation channels, which are represented by the $L_{\mathrm{r}} \times L_{\mathrm{t}}$ complex random matrix $\mathbf{H}$. With $L \triangleq L_{\mathrm{t}} L_{\mathrm{r}}$ denoting the diversity order, the $L$ complex channel coefficients are distributed according to an arbitrary joint PDF $p(\mathbf{H})$ that characterizes the fading. Since the channel is assumed to remain constant during the length of one frame of $K+K_{\mathrm{p}}$ symbols (block fading), the receiver separately observes the $L_{\mathrm{r}} \times K_{\mathrm{c}}$ matrices

$$
\mathbf{R}(k)=\sqrt{E_{\mathrm{s}}} \mathbf{H C}(k)+\mathbf{W}(k)
$$

with $1 \leq k \leq K / K_{\mathrm{c}}$ and the $L_{\mathrm{r}} \times K_{\mathrm{p}}$ matrix

$$
\mathbf{R}_{\mathrm{p}}=\sqrt{E_{\mathrm{p}}} \mathbf{H C}_{\mathrm{p}}+\mathbf{W}_{\mathrm{p}}
$$

where the noise matrices $\mathbf{W}(k)$ and $\mathbf{W}_{\mathrm{p}}$ affecting the transmission of data and pilot symbols, respectively, consist of i.i.d. zeromean (ZM) circularly symmetric complex Gaussian (CSCG) random variables (RVs) with variance $N_{0}$. Because of (3) and 
(6), $E_{\mathrm{s}}$ and $E_{\mathrm{p}}$ in (7) and (8) can be considered as the average data and pilot energy, respectively. In the remainder of this paper, we will omit the block index $k$ for notational convenience.

\section{MiSMATCHED ML RECEIVER}

Using the known pilot matrix $\mathbf{C}_{\mathrm{p}}$ and the corresponding received signal matrix $\mathbf{R}_{\mathrm{p}}$, the receiver can estimate the channel matrix $\mathbf{H}$. Assuming orthogonal pilot sequences, i.e., $\mathbf{C}_{\mathrm{p}} \mathbf{C}_{\mathrm{p}}^{H}=$ $K_{\mathrm{p}} \mathbf{I}_{L_{\mathrm{t}}}$, and LS channel estimation, it is shown in [25] that the channel estimate $\hat{\mathbf{H}}$ is given by

$$
\hat{\mathbf{H}}=\frac{1}{K_{\mathrm{p}} \sqrt{E_{\mathrm{p}}}} \mathbf{R}_{\mathrm{p}} \mathbf{C}_{\mathrm{p}}^{H}
$$

such that $\hat{\mathbf{H}}$ can be decomposed into the following sum of two statistically independent contributions

$$
\hat{\mathbf{H}}=\mathbf{H}+\mathbf{N} \text {. }
$$

The entries of the $L_{\mathrm{r}} \times L_{\mathrm{t}}$ estimation noise matrix $\mathbf{N}=\left[1 /\left(K_{\mathrm{p}} \sqrt{E_{\mathrm{p}}}\right)\right] \mathbf{W}_{\mathrm{p}} \mathbf{C}_{\mathrm{p}}^{H}$ are ZM CSCG RVs, the real and imaginary parts of which have variance $\sigma_{\mathrm{N}}^{2}=N_{0} /\left(2 K_{\mathrm{p}} E_{\mathrm{p}}\right)$ [25]. Due to this fact, when conditioned on $\mathbf{H}$, the estimated channel coefficients $\hat{\mathbf{H}}_{\ell, k}\left(1 \leq \ell \leq L_{\mathrm{r}}, 1 \leq k \leq L_{\mathrm{t}}\right)$ are CSCG $\mathrm{RVs}$ with mean $\mathbf{H}_{\ell, k}$ and variance $2 \sigma_{\mathrm{N}}^{2}$. Increasing the total energy $K_{\mathrm{p}} E_{\mathrm{p}}$ allocated to pilot symbols improves the channel estimate, but also reduces the symbol energy $E_{s}$ available for data transmission. With $E_{\mathrm{b}}$ denoting the average energy per information bit, so that the total energy per frame is constrained to $E_{\text {tot }}=\left(K / K_{\mathrm{c}}\right) N_{\mathrm{s}} \log _{2}(M) E_{\mathrm{b}}$, we have [25]

$$
E_{\mathrm{s}}=\frac{K}{K+\gamma K_{\mathrm{p}}} \rho \log _{2}(M) E_{\mathrm{b}}
$$

where $\gamma \triangleq E_{\mathrm{p}} / E_{\mathrm{S}}, M$, and $\rho \triangleq N_{\mathrm{s}} /\left(L_{\mathrm{t}} K_{\mathrm{c}}\right)$ denote the ratio of $E_{\mathrm{p}}$ to $E_{\mathrm{s}}$, the constellation size, and the code rate, respectively. Note that $E_{\mathrm{s}}$ is a decreasing function of $K_{\mathrm{p}}$.

When the receiver knows the channel matrix $\mathbf{H}$, ML detection is known to be the optimal detection algorithm for the coded data [26, Ch. 7]. In this paper, we consider a mismatched ML receiver that uses the estimated channel $\hat{\mathbf{H}}$ in the same way as an ML receiver would apply $\mathbf{H}$. In this way, the detection algorithm for the code matrix $\mathbf{C}$ is given by

$$
\hat{\mathbf{C}}=\arg \min _{\widetilde{\mathbf{C}}}\left\|\mathbf{R}-\sqrt{E_{\mathrm{S}}} \hat{\mathbf{H}} \widetilde{\mathbf{C}}\right\|_{\mathrm{F}}^{2}
$$

where the minimization is over the valid code matrices $\widetilde{\mathbf{C}}$ satisfying (1). Using (1) and (2), the detection algorithm given by (12) for the information symbols $s_{i}$, reduces to symbol-bysymbol detection

$$
\hat{s}_{i}=\arg \min _{\tilde{s} \in \Psi}\left|u_{i}-\tilde{s}\right|, \quad 1 \leq i \leq N_{\mathrm{s}}
$$

where the minimization is now over the symbols $\tilde{s}$ belonging to the considered constellation $\Psi$ and the decision variables $u_{i}=$ $u_{i, \mathrm{R}}+j u_{i, \mathrm{I}}$, with $u_{i, \mathrm{R}}$ and $u_{i, \mathrm{I}}$ denoting the real and imaginary parts of $u_{i}$, respectively, are given by

$$
u_{i}=\frac{\operatorname{tr}\left(\mathbf{C}_{i}^{H} \hat{\mathbf{H}}^{H} \mathbf{R}+\mathbf{R}^{H} \hat{\mathbf{H}} \mathbf{C}_{i}^{\prime}\right)}{\lambda \sqrt{E_{\mathrm{s}}}\|\hat{\mathbf{H}}\|_{\mathrm{F}}^{2}} .
$$

\section{EXACT BER ANALYSIS}

In this section, we derive exact BER expressions for PAM and square QAM constellations with Gray mapping. Since square $M$-QAM with Gray mapping reduces to $\sqrt{M}$-PAM for both the in-phase and quadrature-phase information bits, the BER computation for PAM follows the same lines as the BER computation for the in-phase bits in case of QAM. Therefore, the BER expressions for PAM are not given explicitly.

For $M$-QAM, the BER of the OSTBC is given by the following expectation:

$$
\mathrm{BER}=\frac{1}{2 N_{\mathrm{s}}} \sum_{i=1}^{N_{\mathrm{s}}} \mathbb{E}\left[\operatorname{BER}_{i, \mathrm{R}}(\mathbf{s}, \mathbf{H}, \hat{\mathbf{H}})+\operatorname{BER}_{i, \mathrm{I}}(\mathbf{s}, \mathbf{H}, \hat{\mathbf{H}})\right]
$$

where $\operatorname{BER}_{i, \mathrm{R}}(\mathbf{s}, \mathbf{H}, \hat{\mathbf{H}})$ and $\operatorname{BER}_{i, \mathrm{I}}(\mathbf{s}, \mathbf{H}, \hat{\mathbf{H}})$ denote the conditional BERs related to the in-phase and quadrature-phase information bits of $s_{i}$, respectively, conditioned on the channel $\mathbf{H}$, the channel estimate $\hat{\mathbf{H}}$ and the symbol vector $\mathbf{s}$. Denoting by $\Psi$ the $M$-QAM constellation, we assume that $\mathbf{s}$ is uniformly distributed over $\Psi^{N_{\mathrm{s}}}$. In general, $\operatorname{BER}_{i, \mathrm{R}}(\mathbf{s}, \mathbf{H}, \hat{\mathbf{H}})$ and $\operatorname{BER}_{i, \mathrm{I}}(\mathbf{s}, \mathbf{H}, \hat{\mathbf{H}})$ are not identical and their value may depend on the index $i$ of the considered symbol $s_{i}$.

Let us consider a QAM symbol $b=b_{\mathrm{R}}+j b_{\mathrm{I}}$, with $b_{\mathrm{R}}$ and $b_{\mathrm{I}}$ denoting the real and imaginary parts of $b$, respectively. $\Psi_{\mathrm{R}}$ and $\Psi_{\mathrm{I}}$ are the sets consisting of the real and imaginary parts of the constellation points, respectively. Referring to the projections of the decision area of $b$ on the real and imaginary axis as the decision regions of $b_{\mathrm{R}}$ and $b_{\mathrm{I}}$, respectively, $\mathrm{BER}_{i, \mathrm{q}}(\mathbf{s}, \mathbf{H}, \hat{\mathbf{H}})$ in (15), with $\mathrm{q}=\mathrm{R}$ or $\mathrm{q}=\mathrm{I}$, can be expressed as

$$
\mathrm{BER}_{i, \mathrm{q}}(\mathbf{s}, \mathbf{H}, \hat{\mathbf{H}})=2 \sum_{b_{\mathrm{q}} \in \Psi_{\mathrm{q}}} \frac{d_{\mathrm{H}}\left(s_{i, \mathrm{q}}, b_{\mathrm{q}}\right)}{\log _{2} M} P_{i, \mathrm{q}}\left(\mathbf{s}, b_{\mathrm{q}}, \mathbf{H}, \hat{\mathbf{H}}\right)
$$

where $d_{\mathrm{H}}\left(s_{i, \mathrm{q}}, b_{\mathrm{q}}\right)$ represents the Hamming distance between the bits allocated to $s_{i, \mathrm{q}}$ and the bits allocated to $b_{\mathrm{q}}$, and $P_{i, \mathrm{q}}\left(\mathbf{s}, b_{\mathrm{q}}, \mathbf{H}, \hat{\mathbf{H}}\right)$ is the probability that $u_{i, \mathrm{q}}$ is located inside the decision area of $b_{\mathrm{q}}$, when $\mathbf{s}, \mathbf{H}$, and $\hat{\mathbf{H}}$ are known. This probability is given by

$$
P_{i, \mathrm{q}}\left(\mathbf{s}, b_{\mathrm{q}}, \mathbf{H}, \hat{\mathbf{H}}\right)=\operatorname{Pr}\left[\hat{s}_{i, \mathrm{q}}=b_{\mathrm{q}} \mid \mathbf{s}, \mathbf{H}, \hat{\mathbf{H}}\right] .
$$

Taking (7) and (1) into account, expanding the decision variable (14) as a summation of a signal $u_{i}^{\prime}=u_{i, \mathrm{R}}^{\prime}+j u_{i, \mathrm{I}}^{\prime}$, with $u_{i, \mathrm{R}}^{\prime}$ and $u_{i, \mathrm{I}}^{\prime}$ denoting the real and imaginary parts of $u_{i}^{\prime}$, respectively, and a noise contribution, yields

$$
u_{i}=u_{i}^{\prime}+\frac{\operatorname{tr}\left(\mathbf{C}_{i}^{H} \hat{\mathbf{H}}^{H} \mathbf{W}+\mathbf{W}^{H} \hat{\mathbf{H}} \mathbf{C}_{i}^{\prime}\right)}{\lambda \sqrt{E_{\mathrm{s}}}\|\hat{\mathbf{H}}\|_{\mathrm{F}}^{2}}
$$

where

$$
u_{i}^{\prime} \triangleq \frac{\operatorname{tr}\left(\mathbf{C}_{i}^{H} \hat{\mathbf{H}}^{H} \mathbf{H C}+\mathbf{C}^{H} \mathbf{H}^{H} \hat{\mathbf{H}} \mathbf{C}_{i}^{\prime}\right)}{\lambda\|\hat{\mathbf{H}}\|_{\mathrm{F}}^{2}} .
$$

It follows from (1) that, in general, $u_{i}^{\prime}$ contains a useful term proportional to $s_{i}$ and interference terms containing the data symbols $s_{n}$, with $n \neq i$. The second term in (18) represents ZM CSCG noise, the real and imaginary parts of which have variance $N_{0} /\left(2 \lambda E_{\mathrm{S}}\|\hat{\mathbf{H}}\|_{\mathrm{F}}^{2}\right)$, which is irrespective of $i$. If PCSI 
is available, i.e., $\hat{\mathbf{H}}=\mathbf{H}$, (19) reduces to $u_{i}^{\prime}=s_{i}$ because of (4), indicating that only a useful signal term is present. Due to the rotational symmetry of the $M$-QAM constellation and the uniform distribution of the symbol vector $\mathbf{s}$, it follows that the BERs related to the in-phase and quadrature-phase bits of $s_{i}$ are identical and irrespective of $i$ in case of PCSI.

To further detail (17), we introduce $d_{1}\left(b_{\mathrm{q}}\right)$ and $d_{2}\left(b_{\mathrm{q}}\right)$, which denote the boundaries of the decision area of $b_{\mathrm{q}}$, with $d_{1}\left(b_{\mathrm{q}}\right)<$ $d_{2}\left(b_{\mathrm{q}}\right)$; for outer constellation points, we set $d_{1}\left(b_{\mathrm{q}}\right) \rightarrow-\infty$ or $d_{2}\left(b_{\mathrm{q}}\right) \rightarrow \infty$. In this way, we have

$$
P_{i, \mathrm{q}}\left(\mathbf{s}, b_{\mathrm{q}}, \mathbf{H}, \hat{\mathbf{H}}\right)=Q_{i, \mathrm{q}, 1}-Q_{i, \mathrm{q}, 2}
$$

where the quantities $Q_{i, \mathrm{q}, k}$, with $k=1$ and 2 , are given by

$$
Q_{i, \mathrm{q}, k} \triangleq Q\left\{\sqrt{2 \lambda \frac{E_{\mathrm{s}}}{N_{0}}\|\hat{\mathbf{H}}\|_{\mathrm{F}}^{2}}\left[d_{k}\left(b_{\mathrm{q}}\right)-u_{i, \mathrm{q}}^{\prime}\right]\right\}
$$

and $Q(\cdot)$ denotes the Gaussian $Q$-function [26, eq. (4.1)]. Note from (15)-(21) that the evaluation of the BER requires averaging over $4 L$ real-valued continuous RVs, i.e., the real and imaginary parts of the elements of $\mathbf{H}$ and $\hat{\mathbf{H}}$, and over $N_{\mathrm{s}}$ discrete RVs, i.e., the $N_{\mathrm{s}}$ symbols contained in $\mathbf{s}$.

In order to reduce the computational complexity related to the numerical evaluation of the BER, we will decrease the number of RVs involved in the expectation (15) by using an appropriate coordinate transformation. To this end, we introduce the $2 L \times 1$ real-valued column vectors $\hat{\mathbf{h}}$ and $\mathbf{h}$, which contain all elements of $\hat{\mathbf{H}}$ and $\mathbf{H}$, respectively, as

$$
\begin{gathered}
\hat{\mathbf{h}} \triangleq\left(\hat{\mathbf{h}}_{1, \mathrm{R}}^{T}, \hat{\mathbf{h}}_{1, \mathrm{I}}^{T}, \hat{\mathbf{h}}_{2, \mathrm{R}}^{T}, \hat{\mathbf{h}}_{2, \mathrm{I}}^{T}, \ldots, \hat{\mathbf{h}}_{N_{\mathrm{s}}, \mathrm{R}}^{T}, \hat{\mathbf{h}}_{N_{\mathrm{s}}, \mathrm{I}}^{T}\right)^{T} \\
\mathbf{h} \triangleq\left(\mathbf{h}_{1, \mathrm{R}}^{T}, \mathbf{h}_{1, \mathrm{I}}^{T}, \mathbf{h}_{2, \mathrm{R}}^{T}, \mathbf{h}_{2, \mathrm{I}}^{T}, \ldots, \mathbf{h}_{N_{\mathrm{s}}, \mathrm{R}}^{T}, \mathbf{h}_{N_{\mathrm{s}}, \mathrm{I}}^{T}\right)^{T}
\end{gathered}
$$

with $\hat{\mathbf{h}}_{i, \mathrm{R}}+j \hat{\mathbf{h}}_{i, \mathrm{I}}$ and $\mathbf{h}_{i, \mathrm{R}}+j \mathbf{h}_{i, \mathrm{I}}$ denoting the $i$ th column of $\hat{\mathbf{H}}$ and $\mathbf{H}$, respectively. It can be easily seen that $\|\hat{\mathbf{h}}\|=\|\hat{\mathbf{H}}\|_{\mathrm{F}}$ and $\|\mathbf{h}\|=\|\mathbf{H}\|_{\mathrm{F}}$. Using (22), the coordinate transformation derived in Appendix A shows that the real and imaginary parts of (19) can be reduced to

$$
u_{i, \mathrm{q}}^{\prime}=\frac{1}{\lambda\left(x_{1}^{2}+x_{2}^{2}+z^{2}\right)}\left(\lambda x_{1} s_{i, \mathrm{q}}\|\mathbf{h}\|+x_{2}\left\|\mathbf{M}_{i, \mathrm{q}} \mathbf{h}\right\|\right)
$$

where the $2 L \times 2 L$ matrix $\mathbf{M}_{i, \mathrm{q}}$, given by (71), incorporates the interference from the signal components different from $s_{i, \mathrm{q}}$. When conditioned on $\mathbf{h}$, the RVs $x_{1}, x_{2}$, and $z$ are independent and distributed as follows:

- $x_{1}$ is a Gaussian RV with mean $\|\mathbf{h}\|$ and variance $\sigma_{\mathbf{N}}^{2}$;

- $x_{2}$ is a ZM Gaussian RV with variance $\sigma_{\mathrm{N}}^{2}$;

- $z / \sigma_{\mathbf{N}}$ is distributed according to the chi-distribution with $2 L-2$ degrees of freedom [27].

Hence, by substituting $u_{i, q}^{\prime}$ and $\|\hat{\mathbf{H}}\|_{\mathrm{F}}^{2}$ in (21) by (23) and (74), respectively, the conditional BER given by (16) can be rewritten as a function that depends on the actual channel $\mathbf{H}$ through the random vector $\mathbf{h}$ and on the estimated channel $\hat{\mathbf{H}}$ through only three RVs: $x_{1}, x_{2}$, and $z$; we denote this function by $\mathrm{B}_{i, \mathrm{q}, 1}\left(\mathbf{s}, \mathbf{h}, x_{1}, x_{2}, z\right)$. Note that the dependence on $\mathbf{h}$ is only through $\|\mathbf{h}\|$ and $\left\|\mathbf{M}_{i, \mathrm{q}} \mathbf{h}\right\|$, with $\mathbf{M}_{i, \mathrm{q}}$ depending on $\mathbf{s}$. Due to this substitution, the BER expression given by (15) reduces to

$$
\begin{aligned}
\mathrm{BER}=\frac{1}{2 N_{\mathrm{s}}} \sum_{i=1}^{N_{\mathrm{s}}} \mathbb{E}\left[\mathrm{B}_{i, \mathrm{R}, 1}\left(\mathbf{s}, \mathbf{h}, x_{1}, x_{2}, z\right)\right. \\
\left.+\mathrm{B}_{i, \mathrm{I}, 1}\left(\mathbf{s}, \mathbf{h}, x_{1}, x_{2}, z\right)\right]
\end{aligned}
$$

which is an expectation over $2 L+3$ real-valued continuous RVs, i.e., the $2 L$ components of $\mathbf{h}, x_{1}, x_{2}$, and $z$, and $N_{\mathrm{s}}$ discrete $\mathrm{RVs}$, i.e., the components of $\mathbf{s}$.

In the case of square OSTBCs, i.e., $L_{\mathrm{t}}=K_{\mathrm{c}}$, the BER expression can be considerably simplified. For these OSTBCs, it is shown in Appendix B that the magnitude of $\mathbf{M}_{i, \mathrm{q}} \mathbf{h}$ used in (23) is given by

$$
\left\|\mathbf{M}_{i, \mathrm{q}} \mathbf{h}\right\|=\lambda\|\mathbf{h}\| \sqrt{\|\mathbf{s}\|^{2}-s_{i, \mathrm{q}}^{2}}
$$

Hence, by substituting $\left\|\mathbf{M}_{i, \mathrm{q}} \mathbf{h}\right\|$ in $\mathrm{B}_{i, \mathbf{q}, 1}\left(\mathbf{s}, \mathbf{h}, x_{1}, x_{2}, z\right)$ by (25), $\mathrm{B}_{i, q, 1}\left(\mathbf{s}, \mathbf{h}, x_{1}, x_{2}, z\right)$ can be rewritten as a function that depends on $\mathbf{h}$ through only the norm $\|\mathbf{h}\|$ of the channel vector; we denote this function by $\mathrm{B}_{i, \mathrm{q}, 2}\left(\mathbf{s},\|\mathbf{h}\|, x_{1}, x_{2}, z\right)$. It follows from (25) that, for square OSTBCs, the dependence of (23) on $i$ and $\mathrm{q}$ is through $s_{i, \mathrm{q}}$ only. Since the statistical properties of $s_{i, \mathrm{q}}$ depend neither on $i$ nor on $\mathrm{q}$, the BERs related to the in-phase and quadrature-phase bits of $s_{i}$ are identical and irrespective of $i$, such that (24) reduces to

$$
\mathrm{BER}=\mathbb{E}\left[\mathrm{B}_{i, \mathrm{q}, 2}\left(\mathbf{s},\|\mathbf{h}\|, x_{1}, x_{2}, z\right)\right]
$$

which involves the expectation over only 4 real-valued continuous RVs, i.e., the norm $\|\mathbf{h}\|$ of the channel vector $\mathbf{h}, x_{1}, x_{2}$, and $z$, and $N_{\mathrm{s}}$ discrete RVs, i.e., the components of $\mathbf{s}$.

\section{APPROXIMATE BER ANALYSIS}

In this section, we present a simple approximation of the BER for high signal-to-noise ratio (SNR). To this end, we treat the interference caused by the symbols in the OSTBC matrix as additional white Gaussian noise. With $\hat{\mathbf{h}}_{\mathrm{v}} \triangleq \operatorname{vec}(\hat{\mathbf{H}}), \mathbf{h}_{\mathrm{v}} \triangleq$ $\operatorname{vec}(\mathbf{H})$, and $\mathbf{n}_{\mathrm{v}} \triangleq \operatorname{vec}(\mathbf{N})$, it follows from (10) that the estimated channel vector $\hat{\mathbf{h}}_{\mathrm{v}}$ is given by $\hat{\mathbf{h}}_{\mathrm{v}}=\mathbf{h}_{\mathrm{v}}+\mathbf{n}_{\mathrm{v}}$, where the elements of $\mathbf{n}_{\mathrm{v}}$ are i.i.d. ZM CSCG RVs with variance $2 \sigma_{\mathbf{N}}^{2}$. Let us expand $\mathbf{h}_{\mathrm{v}}$ as

$$
\mathbf{h}_{\mathrm{v}}=\mathbf{A} \hat{\mathbf{h}}_{\mathrm{v}}+\boldsymbol{\delta}
$$

where the $L \times L$ matrix $\mathbf{A}$ is chosen in such way that both terms in the sum are uncorrelated. Taking (10) into account, it can be shown that $\mathbf{A}$ is given by

$$
\mathbf{A}=\left(\boldsymbol{\mathcal { R }}_{\mathrm{h}}+\frac{N_{0}}{K_{\mathrm{p}} E_{\mathrm{p}}} \mathbf{I}_{L}\right)^{-1} \boldsymbol{\mathcal { R }}_{\mathrm{h}}
$$

where $\boldsymbol{R}_{\mathrm{h}} \triangleq \mathbb{E}\left[\mathbf{h}_{\mathrm{v}} \mathbf{h}_{\mathrm{v}}^{H}\right]$ is the covariance matrix of the channel vector $\mathbf{h}_{\mathrm{v}}$. In general, the elements of $\boldsymbol{\delta}$ are not Gaussian (except 
for Rayleigh fading); they are correlated with covariance matrix $\boldsymbol{\mathcal { R }}_{\delta} \triangleq \mathbb{E}\left[\boldsymbol{\delta} \boldsymbol{\delta}^{H}\right]$, given by

$$
\boldsymbol{\mathcal { R }}_{\delta}=\frac{N_{0}}{K_{\mathrm{p}} E_{\mathrm{p}}}\left(\boldsymbol{\mathcal { R }}_{\mathrm{h}}+\frac{N_{0}}{K_{\mathrm{p}} E_{\mathrm{p}}} \mathbf{I}_{L}\right)^{-1} \boldsymbol{\mathcal { R }}_{\mathrm{h}} .
$$

Note that the entries of $\delta$ are uncorrelated in case of uncorrelated channels.

For high SNR, i.e., when $K_{\mathrm{p}} E_{\mathrm{p}} / N_{0} \gg 1 / \mathbb{E}\left[\left|\mathbf{H}_{\ell, k}\right|^{2}\right]$ for $1 \leq$ $\ell \leq L_{\mathrm{r}}$ and $1 \leq k \leq L_{\mathrm{t}}$, it follows from (28) that $\mathbf{A} \approx \mathbf{I}_{L}$. Using this high-SNR approximation, the channel matrix $\mathbf{H}$ can be written as

$$
\mathbf{H} \approx \hat{\mathbf{H}}+\Delta,
$$

where the $L_{\mathrm{r}} \times L_{\mathrm{t}}$ matrix $\boldsymbol{\Delta}$ is determined by the relation $\boldsymbol{\delta}=$ $\operatorname{vec}(\boldsymbol{\Delta})$ and the terms in the sum are uncorrelated. Moreover, according to (29), the elements of $\Delta$ can be considered to be uncorrelated since $\boldsymbol{\mathcal { R }}_{\delta} \approx N_{0} /\left(K_{\mathrm{p}} E_{\mathrm{p}}\right) \mathbf{I}_{L}$. By replacing $\mathbf{H}$ in (19) by (30), the signal $u_{i}^{\prime}$ can be shown to reduce to

$$
u_{i}^{\prime}=s_{i}+\frac{\operatorname{tr}\left(\mathbf{C}_{i}^{H} \hat{\mathbf{H}}^{H} \boldsymbol{\Delta} \mathbf{C}+\mathbf{C}^{H} \boldsymbol{\Delta}^{H} \hat{\mathbf{H}} \mathbf{C}_{i}^{\prime}\right)}{\lambda\|\hat{\mathbf{H}}\|_{\mathrm{F}}^{2}}
$$

where $s_{i}$ denotes the transmitted symbol and the second term in the sum represents symbol interference due to ICSI. When conditioned on $\|\hat{\mathbf{H}}\|_{F}$, the variance of the latter term can be easily shown to be $N_{0} L_{\mathrm{t}} /\left(\lambda E_{\mathrm{s}}\|\hat{\mathbf{H}}\|_{\mathrm{F}}^{2} \gamma K_{\mathrm{p}}\right)$ for square OSTBCs. For nonsquare OSTBCs, the same result can be obtained by making the additional assumption that $\mathbb{E}\left[\left(\mathbf{C}^{H} \mathbf{C}\right)_{m, n}\right]=1 / K_{c} \mathbb{E}\left[\operatorname{tr}\left(\mathbf{C}^{H} \mathbf{C}\right)\right] \delta_{m-n}$, i.e., $\mathbb{E}\left[\left(\mathbf{C}^{H} \mathbf{C}\right)\right]$ is approximated by a diagonal matrix with identical diagonal elements given by the average of the diagonal elements of $\mathbb{E}\left[\left(\mathbf{C}^{H} \mathbf{C}\right)\right]$. Taking (18) and (31) into account, it follows that, for high SNR, the decision variable of the mismatched receiver can be written as $u_{i}=s_{i}+w_{i}$, where the disturbance term $w_{i}$ is assumed to be CSCG and has variance $N_{0}\left[1+L_{\mathrm{t}} /\left(\gamma K_{\mathrm{p}}\right)\right] /\left(\lambda E_{\mathrm{s}}\|\hat{\mathbf{H}}\|_{\mathrm{F}}^{2}\right)$. Hence, the BER reduces to an expectation over the Frobenius norm $\|\hat{\mathbf{H}}\|_{F}$ of the estimated channel. Note that, in case of PCSI, the variance of $w_{i}$ is given by $N_{0} /\left(\lambda E_{\mathrm{s}}\|\mathbf{H}\|_{\mathrm{F}}^{2}\right)$. Assuming that the PDF of $\|\hat{\mathbf{H}}\|_{\mathrm{F}}$ approaches the PDF of $\|\mathbf{H}\|_{\mathrm{F}}$ for high SNR, it is now easily seen that, for given $E_{\mathrm{s}} / N_{0}$, the BER for OSTBCs with ICSI can be approximated as the BER in case of PCSI, shifted over an amount of $10 \log _{10}\left[1+L_{\mathrm{t}} /\left(\gamma K_{\mathrm{p}}\right)\right] \mathrm{dB}$.

\section{Evaluation OF THE BER}

In Section IV, we have provided an exact analysis of the BER for OSTBCs on fading channels with ICSI, regardless of the fading distribution. In particular, it has been shown that for any OSTBC, the BER can be written as an expectation over $2 L+3$ real-valued continuous RVs and $N_{\mathrm{s}}$ discrete RVs, as can be seen from (24). Moreover, for square OSTBCs, the BER can be further reduced to an expectation over only 4 real-valued continuous RVs and $N_{\mathrm{s}}$ discrete RVs, as can be seen from (26).

In this section, we deal with the efficient and accurate numerical evaluation of the exact BER expressions (24) and (26). To this end, two numerical integration techniques will be envisaged: the quadrature rule [28, Sec. 4.1] and Monte-Carlo integration [28, Sec. 7.7] with importance sampling [28, Sec. 7.9.1]. Firstly, however, we give a brief description of how the BER can also be obtained by straightforward Monte Carlo simulations and why this method is not to be preferred in the case of OSTBCs.

\section{A. Monte Carlo Simulations}

In order to obtain the BER by Monte Carlo simulations, the input RVs of the system, i.e., the $L$ complex-valued entries of the channel matrix $\mathbf{H}$, the data symbols in the code matrix $\mathbf{C}$, and the noise matrices in (7) and (8), need to be generated repeatedly according to their corresponding distributions. For each set of input RVs, the receiver estimates the channel according to (9) and detects the information symbols from the received signal matrix according to (13). Finally, the BER is obtained by averaging the ratio of the number of bit errors to the total number of bits within one frame over the different simulation runs. The accuracy of the resulting BER can be improved by increasing the number of simulations. Clearly, the smaller the average number of bit errors occurring per simulation, the larger the number of required simulations will be to obtain a certain accuracy. However, as OSTBCs may achieve very low BERs, even at moderate SNR, extremely long simulation times are usually necessary and, moreover, the required simulation time will increase dramatically with the SNR. Therefore, Monte Carlo simulations are, in general, inappropriate for accurate and efficient BER computations of OSTBCs.

\section{B. Efficient Evaluation of (24) and (26)}

By numerically evaluating (24) and (26), the BER for OSTBCs can be efficiently obtained with a computation time that increases only very slowly with the SNR. In this section, we briefly describe the quadrature rule and Monte Carlo integration with importance sampling, point out their benefits and limitations, and apply them to evaluate (24) and (26).

Let us represent (24) and (26) by the following generic expectation:

$$
\mathrm{BER}=\mathbb{E}_{p}[\mathrm{~B}(\mathbf{v})]
$$

where $\mathrm{B}(\mathbf{v})$ is a function of a random vector $\mathbf{v}=\left(\mathbf{s}^{T}, \mathbf{u}^{T}\right)^{T}$ consisting of the symbol vector $\mathbf{s}$ and a random column vector u. Depending on whether (24) or (26) is considered, we define $\mathbf{u} \triangleq\left(\mathbf{h}^{T}, x_{1}, x_{2}, z\right)^{T}$ and $\mathrm{B}(\mathbf{v}) \triangleq\left(1 / 2 N_{\mathrm{s}}\right) \sum_{i=1}^{N_{\mathrm{s}}}\left[\mathrm{B}_{i, \mathrm{R}, 1}(\mathbf{v})+\right.$ $\left.\mathrm{B}_{i, \mathrm{I}, 1}(\mathbf{v})\right]$, or $\mathbf{u} \triangleq\left(\|\mathbf{h}\|, x_{1}, x_{2}, z\right)^{T}$ and $\mathrm{B}(\mathbf{v}) \triangleq \mathrm{B}_{i, \mathrm{q}, 2}(\mathbf{v})$, respectively, with $\mathrm{q}=\mathrm{R}$ or $\mathrm{q}=\mathrm{I}$. In (32), the subscript $p$ refers to the joint PDF $p(\mathbf{v})=p(\mathbf{s}) p(\mathbf{u})$ of $\mathbf{v}$, with $\mathbf{s}$ being uniformly distributed over $\Psi^{N_{\mathrm{s}}}$. In this way, the expectations (24) and (26) are defined by the following sum of a $J$-fold integral, with $J$ denoting the dimension of $\mathbf{u}$,

$$
\mathbb{E}_{p}[\mathrm{~B}(\mathbf{v})] \triangleq \frac{1}{M^{N_{\mathrm{s}}}} \sum_{\mathbf{s} \in \Psi^{N_{\mathrm{s}}}} \int_{\mathbf{u}} \mathrm{B}(\mathbf{s}, \mathbf{u}) p(\mathbf{u}) \mathrm{d} \mathbf{u} .
$$

Note that the computational complexity associated with the summation in (33) is proportional to $M^{N_{\mathrm{s}}}$, which increases 
prohibitively for large constellation size $M$ and/or large number of information symbols $N_{\mathrm{s}}$.

1) The Quadrature Rule: In principle, the $J$-fold integral in (33) can be evaluated by repeatedly applying the quadrature rule to each of the integrals, in which case the $J$-fold integral is replaced by a $J$-fold sum. Assuming that each element of $\mathbf{v}$ takes $I$ values in this sum, the computational complexity for computing the $J$-fold integral in (33) is proportional to $I^{J}$, which is prohibitively large for large $J$. Therefore, numerical integration is only of practical interest for square OSTBCs, where $\mathbf{u} \triangleq\left(\|\mathbf{h}\|, x_{1}, x_{2}, z\right)^{T}$ and $J=4$. In this case, the joint PDF of $\mathbf{u}$ is easily obtained as the product of the PDFs $p\left(x_{1}\|\mid \mathbf{h}\|\right)$, $p\left(x_{2}\right)$, and $p(z)$, which have been specified in Section IV, and the PDF of $\|\mathbf{h}\|$, which can be available as an analytical expression or in the form of a histogram (e.g., as the result of a measurement), since $\|\mathbf{h}\|$ is to be discretized.

2) Monte-Carlo Integration With Importance Sampling: The exponential dependency of the computational complexity related to (33) on $N_{\mathrm{S}}$ and $J$ can be avoided by using Monte Carlo integration combined with importance sampling. In this way, the left-hand side of (33) is approximated by

$$
G_{\mathcal{N}}\left(\left\{\mathbf{v}_{\nu}\right\}\right)=\frac{1}{\mathcal{N}} \sum_{\nu=1}^{\mathcal{N}} \mathrm{B}\left(\mathbf{v}_{\nu}\right) \frac{p\left(\mathbf{v}_{\nu}\right)}{q\left(\mathbf{v}_{\nu}\right)}
$$

where $\left\{\mathbf{v}_{\nu}, \nu=1,2, \ldots, \mathcal{N}\right\}$ are independent samples generated according to a biased PDF $q(\mathbf{v})$. Note that we use the term Monte Carlo integration for both discrete and continuous variables, i.e., the symbol vector $\mathbf{s}$ and the vector $\mathbf{u}$, respectively. In case $q\left(\mathbf{v}_{\nu}\right)=p\left(\mathbf{v}_{\nu}\right)$, (34) reduces to conventional Monte Carlo integration without importance sampling. Defining $e_{\mathcal{N}} \triangleq G_{\mathcal{N}}\left(\left\{\mathbf{v}_{\nu}\right\}\right)-\mathbb{E}_{p}[\mathrm{~B}(\mathbf{v})]$, it can be shown that $\mathbb{E}\left[e_{\mathcal{N}}\right]=0$ and

$$
\mathbb{E}\left[e_{\mathcal{N}}^{2}\right]=\frac{1}{\mathcal{N}}\left\{\mathbb{E}_{q}\left[\left(\frac{\mathrm{B}(\mathbf{v}) p(\mathbf{v})}{q(\mathbf{v})}\right)^{2}\right]-\mathbb{E}_{p}^{2}[\mathrm{~B}(\mathbf{v})]\right\}
$$

where $\mathbb{E}_{q}[\cdot]$ refers to the expectation over the biased PDF $q(\mathbf{v})$. It follows from (35) that $\mathbb{E}\left[e_{\mathcal{N}}^{2}\right]$ can be made arbitrarily small by taking $\mathcal{N}$ sufficiently large. However, the smaller the second factor in (35), the smaller is the value of $\mathcal{N}$ required to achieve a certain value of $\mathbb{E}\left[e_{\mathcal{N}}^{2}\right]$. By making a judicious choice of $q(\mathbf{v})$, we try to minimize this factor, so that reasonably large values of $\mathcal{N}$ yield very good accuracy. It can be easily verified that $q(\mathbf{v})=\mathrm{B}(\mathbf{v}) p(\mathbf{v}) / \mathbb{E}_{p}[\mathrm{~B}(\mathbf{v})]$ is the optimum biased PDF, as it yields $\mathbb{E}\left[e_{\mathcal{N}}^{2}\right]=0$. However, this choice is not practical, since the optimum $q(\mathbf{v})$ depends on the unknown $\mathbb{E}_{p}[\mathrm{~B}(\mathbf{v})]$. Nevertheless, the optimum $q(\mathbf{v})$ inspires us to take

$$
q(\mathbf{v})=\mathrm{B}_{\mathrm{app}}(\mathbf{v}) p(\mathbf{v}) / \mathbb{E}_{p}\left[\mathrm{~B}_{\mathrm{app}}(\mathbf{v})\right]
$$

where $\mathrm{B}_{\mathrm{app}}(\mathbf{v})$ is a suitable approximation of $\mathrm{B}(\mathbf{v})$, i.e., it is chosen in such way that the resulting $q(\mathbf{v})$ allows us to easily generate i.i.d. vectors $\left\{\mathbf{v}_{\nu}\right\}$.

Let us apply Monte Carlo integration with importance sampling for evaluating the BER in the case of nonsquare OSTBCs, where $\mathbf{v} \triangleq\left(\mathbf{s}^{T}, \mathbf{h}^{T}, x_{1}, x_{2}, z\right)^{T}$. For many fading distributions, the vector $\mathbf{h}$ can easily be generated as a transformation $\mathbf{h}=$ $\phi(\boldsymbol{\gamma})$ of a vector $\boldsymbol{\gamma}$ of auxiliary RVs, distributed according to a joint $\operatorname{PDF} p(\boldsymbol{\gamma})$ which is such that the PDF of $\mathbf{h}=\phi(\boldsymbol{\gamma})$ is the desired distribution of the real and imaginary parts of the channel coefficients contained in $\mathbf{h}$. Therefore, we redefine $\mathbf{v}$ in terms of the vector of auxiliary RVs instead of the channel vector, i.e., $\mathbf{v} \triangleq\left(\mathbf{s}^{T}, \boldsymbol{\gamma}^{T}, x_{1}, x_{2}, z\right)^{T}$, such that

$$
\begin{array}{r}
\mathrm{B}(\mathbf{v}) \triangleq \frac{1}{2 N_{\mathrm{s}}} \sum_{i=1}^{N_{\mathrm{s}}}\left\{\mathrm{B}_{i, \mathrm{R}, 1}\left[\mathbf{s}, \phi(\boldsymbol{\gamma}), x_{1}, x_{2}, z\right]\right. \\
\left.+\mathrm{B}_{i, \mathrm{I}, 1}\left[\mathbf{s}, \phi(\boldsymbol{\gamma}), x_{1}, x_{2}, z\right]\right\}
\end{array}
$$

and

$$
p(\mathbf{v})=p(\mathbf{s}) p\left(x_{1}\|\mathbf{h}\|=\|\phi(\boldsymbol{\gamma})\|\right) p\left(x_{2}\right) p(z) p(\boldsymbol{\gamma}) .
$$

In order to identify an approximate $\mathrm{B}_{\mathrm{app}}(\mathbf{v})$ in (36), we will consider $\mathrm{B}(\mathbf{v})$ assuming PCSI, in which case $u_{i, \mathrm{R}}^{\prime}$ from (23) is the sum of $s_{i, \mathrm{R}}$ and a noise term with variance $N_{0} /\left[2 \lambda E_{\mathrm{s}}\|\phi(\boldsymbol{\gamma})\|^{2}\right]$. Denoting by $2 d$ the distance between adjacent QAM constellation points, we approximate the conditional BER assuming PCSI by the following simple expression

$$
\operatorname{B}_{\mathrm{PCSI}}(\mathbf{v}) \approx Q\left(\sqrt{\frac{2 \lambda E_{\mathrm{s}} d^{2}\|\phi(\boldsymbol{\gamma})\|^{2}}{N_{0}}}\right) .
$$

Considering the bound $Q(x) \leq(1 / 2) \exp \left(-x^{2} / 2\right)$, we select

$$
\mathrm{B}_{\mathrm{app}}(\mathbf{v})=\frac{1}{2} \exp \left[-\lambda d^{2} \frac{E_{\mathrm{s}}}{N_{0}}\|\phi(\boldsymbol{\gamma})\|^{2}\right] .
$$

Since $\mathrm{B}_{\mathrm{app}}(\mathbf{v})$ depends only on $\boldsymbol{\gamma}$, the corresponding biased PDF (36) is given by

$$
q(\mathbf{v})=p(\mathbf{s}) p\left(x_{1} \mid\|\mathbf{h}\|=\|\phi(\boldsymbol{\gamma})\|\right) p\left(x_{2}\right) p(z) q(\boldsymbol{\gamma})
$$

with

$$
q(\boldsymbol{\gamma})=C p(\boldsymbol{\gamma}) \exp \left[-\lambda d^{2} \frac{E_{\mathrm{s}}}{N_{0}}\|\phi(\boldsymbol{\gamma})\|^{2}\right]
$$

where $C$ is a normalization constant. From (41), it follows that only the joint PDF $q(\boldsymbol{\gamma})$ of the vector $\boldsymbol{\gamma}$ of auxiliary RVs is biased. Hence, $\mathbf{s}, x_{1}, x_{2}$, and $z$ need to be generated according to their respective distributions, whereas the auxiliary RVs are generated according to the biased joint PDF given by (42), which depends on the considered transformation $\phi(\gamma)$.

Note that also a combination of the quadrature rule and Monte Carlo integration can be used. For example, for square OSTBCs, the integral over $\mathbf{u} \triangleq\left(\|\mathbf{h}\|, x_{1}, x_{2}, z\right)^{T}$ can be evaluated using the quadrature rule, whereas the summation over the symbol vector $\mathbf{s}$ can be evaluated through Monte Carlo integration. In order to further reduce the computational complexity related to the evaluation of (24), B(v) can be approximated by retaining only one term in the summation in (37), i.e., $\mathrm{B}_{i, \mathrm{q}, 1}\left[\mathbf{s}, \phi(\gamma), x_{1}, x_{2}, z\right]$, with $\mathrm{q}=\mathrm{R}$ or $\mathrm{q}=\mathrm{I}$, instead of taking the average over all $2 N_{\mathrm{s}}$ terms. In Section VIII, comparisons between BER results obtained using this approximation and computer simulations show that this approximation is very accurate. 


\section{CoRrelated NAKAGAMI- $m$ FAdING Channels}

In this section, we apply the theory shown in Section VI-B for the evaluation of the BER of OSTBCs with ICSI to the particular case of arbitrarily correlated Nakagami- $m$ fading channels.

The Nakagami- $m$ distribution [29] is considered as a versatile statistical distribution that accurately models a variety of fading environments by selecting a proper value for the fading parameter $m \geq 1 / 2$. It includes the Rayleigh $(m=1)$ and the one-sided Gaussian $(m=1 / 2)$ distributions as special cases. Moreover, for $m \rightarrow \infty$, the fading channel converges to an AWGN channel. Denoting by $\alpha$ the magnitude of a complex-valued channel coefficient, its PDF in the case of Nakagami- $m$ fading is given by [26, eq. (2.20)]

$$
p_{\alpha}(r)=\frac{2}{\Gamma(m)}\left(\frac{m}{\Omega}\right)^{m} r^{2 m-1} \exp \left(-\frac{m}{\Omega} r^{2}\right) U(r)
$$

with $\Gamma(\cdot)$ being the Gamma function [30, eq. (8.310/1)], $U(r)$ being the unit step function, and $\Omega=\mathbb{E}\left[\alpha^{2}\right]$ being the average fading power. Equivalently, the fading power $\alpha^{2}$ follows a Gamma distribution with shape parameter $m$ and mean $\Omega$ [26, eq. (2.21)]. Assuming PCSI, it follows from [31] that the BER of an OSTBC operating over $L$ i.i.d. Nakagami- $m$ fading channels is proportional to $\left(E_{\mathrm{b}} / N_{0}\right)^{-m L}$ for large $E_{\mathrm{b}} / N_{0}$, which indicates that the BER performance improves with increasing $m$ and/or diversity order $L$.

In case of a correlated Nakagami fading MIMO channel, the marginal PDF of the magnitude $\alpha_{k, \ell}$ of the channel coefficient $\mathbf{H}_{k, \ell}$ follows (43), with parameters $m_{k, \ell}$ and $\Omega_{k, \ell}$ possibly depending on the indexes $k$ and $\ell$. Stacking the $L=L_{\mathrm{t}} L_{\mathrm{r}}$ fading magnitudes $\alpha_{k, \ell}$ into an $L$-dimensional column vector $\boldsymbol{\alpha}$, such that $\boldsymbol{\alpha}_{k+(\ell-1) L_{\mathrm{r}}}=\alpha_{k, \ell}$, the elements of the $L \times L$ power correlation matrix $\boldsymbol{\Sigma}$ of $\boldsymbol{\alpha}$ are defined as [26, eq. (9.195)]

$$
\Sigma_{i, n} \triangleq \frac{\operatorname{cov}\left(\boldsymbol{\alpha}_{i}^{2}, \boldsymbol{\alpha}_{n}^{2}\right)}{\sqrt{\operatorname{var}\left(\boldsymbol{\alpha}_{i}^{2}\right) \operatorname{var}\left(\boldsymbol{\alpha}_{n}^{2}\right)}}
$$

with $i, n=1,2, \ldots, L$. Using the MIMO channel model proposed in [32], (44) is decomposed as

$$
\Sigma=\Sigma_{\mathrm{t}} \otimes \Sigma_{\mathrm{r}}
$$

where $\Sigma_{\mathrm{t}}$ and $\Sigma_{\mathrm{r}}$ are the $L_{\mathrm{t}} \times L_{\mathrm{t}}$ transmit and $L_{\mathrm{r}} \times L_{\mathrm{r}}$ receive power correlation matrices, respectively, the elements of which are defined as

$$
\left(\Sigma_{\mathrm{t}}\right)_{i, n} \triangleq \frac{\operatorname{cov}\left(\alpha_{l, i}^{2}, \alpha_{l, n}^{2}\right)}{\sqrt{\operatorname{var}\left(\alpha_{l, i}^{2}\right) \operatorname{var}\left(\alpha_{l, n}^{2}\right)}} \quad 1 \leq l \leq L_{\mathrm{r}}
$$

with $i, n=1,2, \ldots, L_{\mathrm{t}}$, and

$$
\left(\boldsymbol{\Sigma}_{\mathrm{r}}\right)_{i, n} \triangleq \frac{\operatorname{cov}\left(\alpha_{i, l}^{2}, \alpha_{n, l}^{2}\right)}{\sqrt{\operatorname{var}\left(\alpha_{i, l}^{2}\right) \operatorname{var}\left(\alpha_{n, l}^{2}\right)}} \quad 1 \leq l \leq L_{\mathrm{t}},
$$

with $i, n=1,2, \ldots, L_{\mathrm{r}}$. According to (46), $\boldsymbol{\Sigma}_{\mathrm{t}}$ and $\boldsymbol{\Sigma}_{\mathrm{r}}$ are independent of the index of the considered receive antenna and transmit antenna, respectively. Also, from the Kronecker model (45), it follows that the normalized covariance between the fading powers $\alpha_{k, \ell}^{2}$ and $\alpha_{i, n}^{2}$ equals $\left(\Sigma_{\mathrm{t}}\right)_{\ell, n}\left(\boldsymbol{\Sigma}_{\mathrm{r}}\right)_{k, i}$.

\section{A. Generating Nakagami-m RVs}

In Section VI-B, we have shown that the expectation over $\mathbf{h}$ in (24) can be efficiently evaluated by means of Monte Carlo integration with importance sampling, provided that the vector $\boldsymbol{\gamma}$ of auxiliary RVs that is used to obtain the channel vector $\mathbf{h}$ is generated according to a proper sampling distribution given by (42). In this section, we give a brief overview of how correlated Nakagami- $m$ RVs can be generated from auxiliary RVs and how (42) is obtained for integer and identical fading parameters, i.e., $m_{\ell}=m$, for $\ell=1,2, \ldots, L$.

Usually, arbitrarily correlated Nakagami-m RVs are generated from either Gamma RVs [33]-[35] or Gaussian RVs [36]-[40]. An efficient method for generating bivariate Nakagami- $m$ samples based on the rejection method is given in [41], for arbitrary values of $m \geq 0.8$. For integer and identical fading parameters, i.e., $m_{\ell}=m, \forall \ell$, it is shown in [39] that $L$ correlated Nakagami- $m$ RVs $\boldsymbol{\alpha}_{\ell}$, can be obtained from $2 m$ i.i.d. real-valued ZM Gaussian random vectors $\mathbf{y}_{k}=\left(y_{k, 1}, y_{k, 2}, \ldots, y_{k, L}\right)^{T}$, with $k=1,2, \ldots, 2 m$. In particular, by defining

$$
\boldsymbol{\alpha}_{\ell}^{2} \triangleq \sum_{k=1}^{2 m} y_{k, \ell}^{2},
$$

it is readily verified that $\boldsymbol{\alpha}_{\ell}$ 's are correlated Nakagami- $m$ RVs with $\mathbb{E}\left[\boldsymbol{\alpha}_{\ell}^{2}\right]=\Omega_{\ell}$ and power correlation matrix $\boldsymbol{\Sigma}$, if the covariance matrix $\mathbf{Q}=\mathbb{E}\left[\mathbf{y}_{k} \mathbf{y}_{k}^{T}\right]$ of the column vectors $\mathbf{y}_{k}$ is given by

$$
\mathrm{Q}=\frac{1}{2 m} \sqrt{\mathbf{\Omega}} \Sigma_{\mathbf{G}} \sqrt{\mathbf{\Omega}}
$$

where the $L \times L$ diagonal matrix $\boldsymbol{\Omega}$ is given by $\boldsymbol{\Omega}=$ $\operatorname{diag}\left\{\Omega_{1}, \Omega_{2}, \ldots, \Omega_{L}\right\}$ and $\Sigma_{\mathbf{G}}=\sqrt{\Sigma}$. The correlated Gaussian entries of the $\mathbf{y}_{k}$ 's can simply be obtained as $\mathbf{y}_{k}=\mathbf{P x}_{k}$, where the $L$-dimensional column vector $\mathbf{x}_{k}$ consists of i.i.d. Gaussian RVs with ZM and unit variance and the $L \times L$ matrix $\mathbf{P}$ results from the Cholesky decomposition [42, p. 559] of the covariance matrix $\mathbf{Q}$, i.e., $\mathbf{Q}=\mathbf{P P}^{T}$.

Let us select $\boldsymbol{\gamma}=\left(\boldsymbol{\theta}^{T}, \mathbf{y}^{T}\right)^{T}$ as the vector of auxiliary RVs, where $\boldsymbol{\theta}$ contains the phases of the $L$ channel coefficients and $\mathbf{y}=\left(\mathbf{y}_{1}^{T}, \mathbf{y}_{2}^{T}, \ldots, \mathbf{y}_{2 m}^{T}\right)^{T}$. Taking into account that $\mathbf{h}=\phi(\boldsymbol{\gamma})$ and that the $L$ channel coefficient magnitudes $\boldsymbol{\alpha}_{\ell}$ are obtained from $\mathbf{y}$ according to (47), we have $\|\mathbf{h}\|^{2}=\|\phi(\boldsymbol{\gamma})\|^{2}=\|\boldsymbol{\alpha}\|^{2}=$ $\|\mathbf{y}\|^{2}$. Hence, with $q(\boldsymbol{\theta}, \mathbf{y})=p(\boldsymbol{\theta} \mid \mathbf{y}) q(\mathbf{y})$, it follows from (42) that the biased joint PDF of $\mathbf{y}$ is given by

$$
q(\mathbf{y})=C p(\mathbf{y}) \exp \left(-\lambda d^{2} \frac{E_{\mathrm{s}}}{N_{0}}\|\mathbf{y}\|^{2}\right) .
$$

Because $p(\mathbf{y})$ is the joint PDF of $2 m$ i.i.d. ZM Gaussian vectors $\left\{\mathbf{y}_{k}\right\}$, each having a covariance matrix $\mathbf{Q}$ given by (48), it follows from (49) that $q(\mathbf{y})$ is a similar PDF, but now the vectors $\left\{\mathbf{y}_{k}\right\}$ have a covariance matrix $\mathbf{Q}^{\prime}$ given by

$$
\mathbf{Q}^{\prime}=\mathbf{Q}\left(\mathbf{I}_{L}+2 \lambda d^{2} \frac{E_{\mathrm{s}}}{N_{0}} \mathbf{Q}\right)^{-1}
$$


Moreover, using (49) and (50), it can be easily shown that the ratio $p(\mathbf{v}) / q(\mathbf{v})$ to be used in (34) depends only on $\mathbf{y}$ and is given by

$$
\frac{p(\mathbf{v})}{q(\mathbf{v})}=\frac{\exp \left(\lambda d^{2} \frac{E_{\mathrm{s}}}{N_{0}}\|\mathbf{y}\|^{2}\right)}{\left[\operatorname{det}\left(\mathbf{I}_{L}+2 \lambda d^{2} \frac{E_{\mathrm{s}}}{N_{0}} \mathbf{Q}\right)\right]^{m}}
$$

\section{B. PDF of the Nakagami-m Channel Norm}

For square OSTBCs, we have shown in Section VI-B that the expectation over the channel norm $\|\mathbf{h}\|$ in (26) can be evaluated by means of the quadrature rule, provided that the PDF of $\|\mathbf{h}\|$ is available as an analytical expression or in the form of a histogram. In this section, we give a brief overview of the available literature on how the PDF of $\|\mathbf{h}\|^{2}$, which we denote by $p_{\|\mathbf{h}\|^{2}}(x)$, can be analytically obtained for correlated Nakagami- $m$ channels. From this PDF, the PDF of $\|\mathbf{h}\|$ is easily obtained using a standard RVs transformation.

In the past, different approaches have been presented for deriving analytical expressions for the distribution of $\|\mathbf{h}\|^{2}$ in the case of arbitrarily correlated Nakagami- $m$ fading channels, e.g., see [33]-[39] and references therein. Analytical expressions for the moment generating function (MGF) of $\|\mathbf{h}\|^{2}$ have been derived for integer $m_{\ell}=m, \forall \ell$, [36], [37], [39], integer $m_{\ell}$ [38] and arbitrary $m_{\ell}$ [35]. Although the obtained expressions in [36], [37], [39] can be straightforwardly used for the derivation of $p_{\|\mathbf{h}\|^{2}}(x)$, this seems complicated using the MGF expression presented in [38] and rather difficult with that in [35]. On the other hand, the PDF-based approach has been used for deriving the distribution of $\|\mathbf{h}\|^{2}$ for arbitrary $m_{\ell}=m, \forall \ell$, [33] and for integer $m_{\ell}$ with the restriction that $\Omega_{\ell} / m_{\ell} \neq \Omega_{k} / m_{k}$ if $k \neq \ell$ [34].

For integer and identical $m_{\ell}=m, \forall \ell$, and $\mathbb{E}\left[\boldsymbol{\alpha}_{\ell}^{2}\right]=\Omega_{\ell}$, the PDF of $\|\mathbf{h}\|^{2}$ is given by [36], [37]

$$
p_{\|\mathbf{h}\|^{2}}(x)=\sum_{i=1}^{\kappa} \sum_{q=1}^{c_{i} m} D_{i, q} x^{q-1} \exp \left(-\frac{x}{2 \lambda_{i}}\right) U(x)
$$

where $\lambda_{i}$ 's, $i=1,2, \ldots, \kappa$, are the distinct eigenvalues of $\mathbf{Q}$ given by (48), with corresponding algebraic multiplicities $c_{i}$. In (52), the parameters $D_{i, q}$ are given by

$$
D_{i, q}=\left.\frac{\left(2 \lambda_{i}\right)^{-c_{i} m}}{(q-1) !\left(c_{i} m-q\right) !}\left[\frac{\mathrm{d}^{c_{i} m-q}}{\mathrm{~d} s^{c_{i} m-q}} \Psi_{i}(s)\right]\right|_{s=-\frac{1}{2 \lambda_{i}}}
$$

where

$$
\Psi_{i}(s)=\prod_{\substack{n=1 \\ n \neq i}}^{\kappa}\left(1+2 \lambda_{n} s\right)^{-c_{n} m} .
$$

Alternatively, by applying a tridiagonal decomposition to $\left(\Sigma_{\mathbf{G}}\right)^{-1}$ for integer $m_{\ell}=m$ and $\Omega_{\ell}=\Omega, \forall \ell, p_{\|\mathbf{h}\|^{2}}(x)$ can be obtained from [39] as fast convergent infinite summations. For arbitrary and identical $m_{\ell}=m, \forall \ell$, the PDF of $\|\mathbf{h}\|^{2}$ can be easily obtained from [33, eq. (5)] as an infinite summation. For arbitrary and nonidentical $m_{\ell}$, the PDF of $\|\mathbf{h}\|^{2}$ is given in [43] as an infinite summation and a good truncation of the PDF's infinite summation is proposed. For i.i.d. Nakagami- $m$ distributed fading envelopes with arbitrary and identical $m_{\ell}=m$ and $\Omega_{\ell}=\Omega, \forall \ell$, the PDF of $\|\mathbf{h}\|$ is shown to be distributed according to the Nakagami- $m$ distribution with parameters $L m$ and $L \Omega$ [44].

\section{NUMERICAL RESULTS}

In this section, BER results are presented for correlated Nakagami- $m$ fading channels, under the assumption that $E_{\mathrm{p}}=E_{\mathrm{s}}$ and that the Kronecker channel model (45) is valid [32], [45], although the analysis is also applicable to arbitrary power correlation matrices. The phases of the channel coefficients are assumed to be uniformly distributed. The accuracy of the curves resulting from the numerical evaluation of the exact BER expressions (24) and (26) using the tools provided in Sections VI-B and VII, is illustrated by means of straightforward Monte Carlo simulation results, which are represented by black dots and are added to some of the figures for relatively high BER. In Figs. 1 and 2, the BER curves resulting from the high-SNR approximation given in Section V are also shown, in order to illustrate their accuracy under different fading circumstances.

\section{A. Square OSTBCs}

Let us consider Alamouti's code $\left(L_{\mathrm{t}}=K_{\mathrm{c}}=N_{\mathrm{s}}=2\right)$, which is given by [1]

$$
\mathcal{H}=\left(\begin{array}{cc}
s_{1} & -s_{2}^{*} \\
s_{2} & s_{1}^{*}
\end{array}\right) .
$$

In order to obtain the BER curves for this OSTBC, we evaluate the expectation over $\|\mathbf{h}\|, x_{1}, x_{2}$, and $z$ in (26) by means of the quadrature rule, with the distribution of $\|\mathbf{h}\|$ being derived from (52) in the case of correlated fading and from (43) in the case of i.i.d. fading; the expectation over $\mathbf{s}$ is exactly obtained by means of a finite summation.

In Fig. 1(a), we show the BER curves for a $2 \times 1$ Alamouti MIMO scheme with LS channel estimation under i.i.d. Nakagami- $m$ fading. Also the approximate BER curves, resulting from treating the symbol interference due to ICSI as white Gaussian noise, are shown in the figure. The data frames consist of $K=40$ coded data symbols and $K_{\mathrm{p}}=4$ pilot symbols per transmit antenna, whereas the symbols belong to a 4-QAM constellation. The BER approximation turns out to be relatively accurate, although it is clearly not asymptotically exact when the fading is not Rayleigh distributed, i.e., for $m>1$. The difference between the Gaussian approximation and the exact result is even larger when BPSK transmission is considered, as shown in Fig. 1(b).

Fig. 2 shows the BER of Alamouti's code under correlated identically distributed (i.d.) Nakagami- $m$ channels with $m=4$ and $\Omega=1$. We assume that there is no antenna correlation at the transmitter side, whereas the correlation between the receive antennas $\left(L_{\mathrm{r}}=3\right)$ can be described by means of a constant correlation model [39] determined by the following power correlation matrix:

$$
\boldsymbol{\Sigma}_{\mathrm{r}}=\left(\begin{array}{ccc}
1 & 0.8 & 0.8 \\
0.8 & 1 & 0.8 \\
0.8 & 0.8 & 1
\end{array}\right)
$$




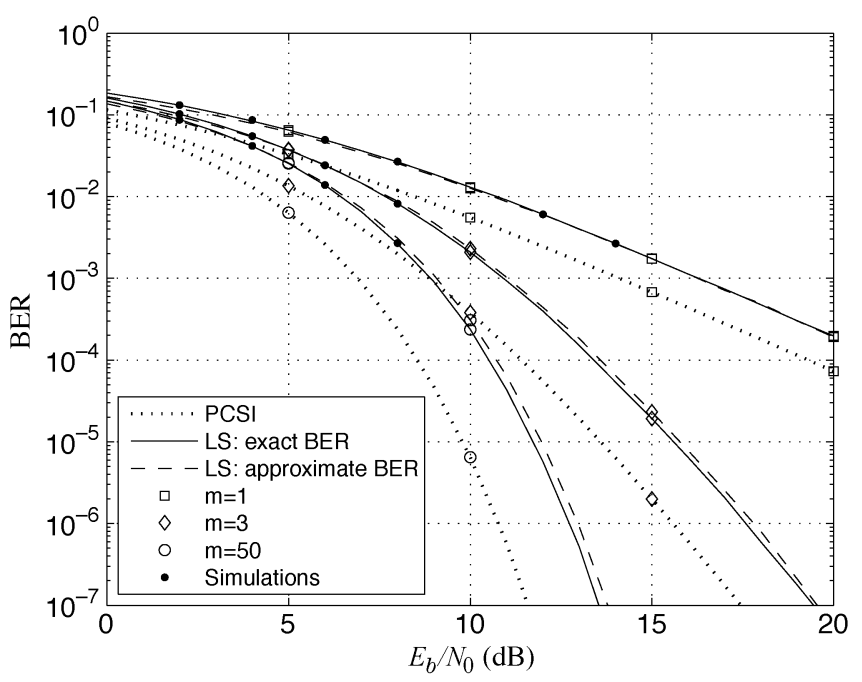

(a)

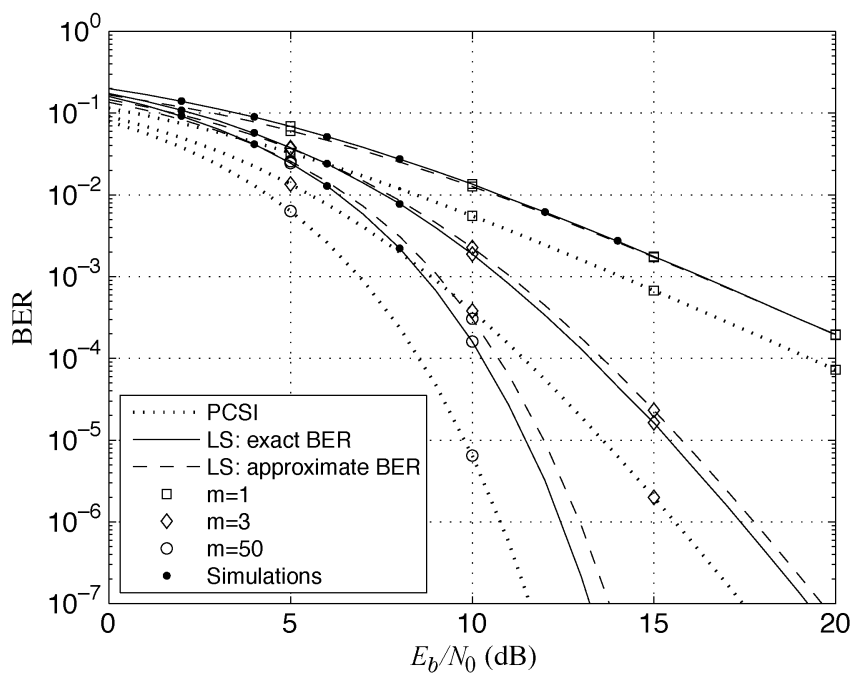

(b)

Fig. 1. Exact and approximate BER versus $E_{\mathrm{b}} / N_{0}$ for a $2 \times 1$ Alamouti scheme with LS channel estimation under i.i.d. Nakagami- $m$ fading. (a) 4-QAM; (b) BPSK.

For $M=\{4,16,64,256\}$, the BER results are shown for a PCSI receiver and a receiver using LS channel estimation with $K=80$ and $K_{\mathrm{p}}=\{2,4,12\}$. For the receiver with ICSI, both the exact and approximate BER curves are displayed. It follows from the figure that the accuracy of the approximate BER curves depends on different parameters, such as the constellation size and the number of pilot symbols $K_{\mathrm{p}}$. The larger $K_{\mathrm{p}}$ is, the smaller the asymptotic difference will be between the approximate and the exact BER curves. This is due to the fact that for large $K_{\mathrm{p}}$, the PDFs of $\|\hat{\mathbf{H}}\|_{\mathrm{F}}$ and $\|\mathbf{H}\|_{\mathrm{F}}$ are very similar such that the approximation from Section $\mathrm{V}$ is quite accurate.

Fig. 3 displays the performance curves resulting from the exact BER expression (26) for Alamouti's code along with 4-QAM signaling, operating over correlated i.d. Nakagami-m channels with $\Omega=1$ and $L_{\mathrm{r}}=3$. The results are shown for both a PCSI receiver and a receiver using LS channel estimation with $K=200$ and $K_{\mathrm{p}}=20$, for $m=\{1,3\}$, and for different correlation models. Assuming that no antenna correlation occurs at the transmitter side, the following correlation models have been considered at the receiver side:

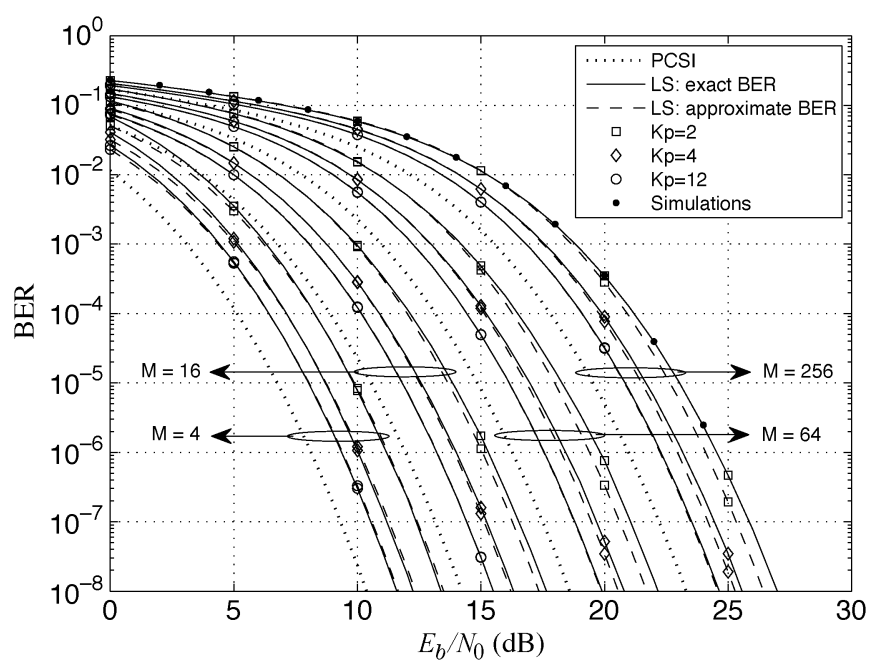

Fig. 2. Exact and approximate BER versus $E_{\mathrm{b}} / N_{0}$ for a $2 \times 3$ Alamouti scheme with LS channel estimation under correlated i.d. Nakagami- $m$ fading, with $K=80, K_{\mathrm{p}}=\{2,4,12\}$, and $M=\{4,16,64,256\}$.

i) uncorrelated fading (unc), with $\boldsymbol{\Sigma}_{\mathrm{r}}=\mathbf{I}_{L_{\mathrm{r}}}$;

ii) a linear antenna array (lin), the configuration of which is depicted in [37, Fig. 4(b)], with power correlation matrix $\Sigma_{\mathrm{r}}$ given by [37, eq. (38)]

$$
\Sigma_{\mathrm{r}}=\left(\begin{array}{ccc}
1 & 0.795 & 0.605 \\
0.795 & 1 & 0.795 \\
0.605 & 0.795 & 1
\end{array}\right)
$$

iii) a triangular antenna array (tri), the configuration of which is depicted in [37, Fig. 4(a)], with power correlation matrix $\Sigma_{\text {r }}$ given by [37, eq. (37)]

$$
\Sigma_{\mathrm{r}}=\left(\begin{array}{ccc}
1 & 0.727 & 0.913 \\
0.727 & 1 & 0.913 \\
0.913 & 0.913 & 1
\end{array}\right)
$$

In Fig. 3, it is shown how $m$, ICSI, and antenna correlation model affect the BER performance of Alamouti's code. As it is expected, the BER performance improves when $m$ increases. As compared to the case of PCSI and zero correlation, both ICSI and antenna correlation degrade the BER through a horizontal shift of the BER curve for large $E_{\mathrm{b}} / N_{0}$, indicating that, for the correlations considered, the relation $\mathrm{BER} \propto\left(E_{\mathrm{b}} / N_{0}\right)^{-m L}$ still holds at large $E_{\mathrm{b}} / N_{0}$. Note that antenna correlation has no significant impact on the BER degradation caused by ICSI only, and that for highly correlated channels, e.g., the triangular correlation model, the BER degradation as compared to zero correlation is much larger than the degradation due to ICSI only.

\section{B. Nonsquare OSTBCs}

Let us consider the $3 \times 4 \operatorname{OSTBC}\left(L_{\mathrm{t}}=N_{\mathrm{s}}=3, K_{\mathrm{c}}=4\right)$ given by [2, eq. (39)]

$$
\mathcal{G}=\sqrt{\frac{4}{3}}\left(\begin{array}{cccc}
s_{1} & -s_{2}^{*} & \frac{s_{3}^{*}}{\sqrt{2}} & \frac{s_{3}^{*}}{\sqrt{2}} \\
s_{2} & s_{1}^{*} & \frac{s_{3}^{*}}{\sqrt{2}} & -\frac{s_{3}^{*}}{\sqrt{2}} \\
\frac{s_{3}}{\sqrt{2}} & \frac{s_{3}}{\sqrt{2}} & \frac{-s_{1}-s_{1}^{*}+s_{2}-s_{2}^{*}}{2} & \frac{s_{2}+s_{2}^{*}+s_{1}-s_{1}^{*}}{2}
\end{array}\right)
$$




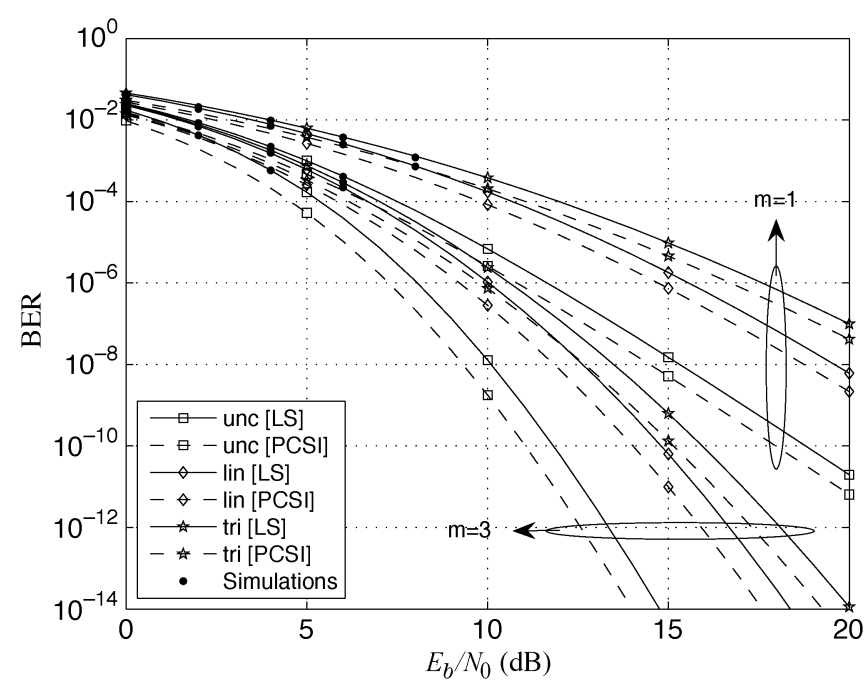

Fig. 3. BER versus $E_{\mathrm{b}} / N_{0}$ for Alamouti's code with 4-QAM signaling and LS channel estimation under correlated i.d. Nakagami- $m$ fading channels with $m=\{1,3\}$ and $\Omega=1$, for uncorrelated (unc), linear (lin), and triangular (tri) correlation matrices at the receiver side $\left(L_{\mathrm{r}}=3\right)$.

where the scaling factor $\sqrt{4 / 3}$ is applied in order that (59) satisfies (2). The BER curves for this OSTBC are obtained by evaluating the expectation over $\mathbf{s}, \mathbf{h}, x_{1}, x_{2}$, and $z$ in (24) by means of Monte Carlo simulation with importance sampling. In (34), however, $\mathrm{B}(\mathbf{v})$ is approximated by retaining only the term $\mathrm{B}_{1, \mathrm{R}, 1}\left[\mathbf{s}, \phi(\gamma), x_{1}, x_{2}, z\right]$ in the summation in (37). Moreover, the auxiliary Gaussian RVs yielding the channel coefficients are generated according to (49).

Fig. 4 shows BER performance evaluation curves in case of a Nakagami- $m$ MIMO channel with $m=2$ that is recovered through LS channel estimation with $K=200$ and $K_{\mathrm{p}}=16$. The power correlation matrix $\Sigma_{\mathrm{t}}$ at the transmitter side is given by (57), whereas the power correlation matrix $\Sigma_{\mathrm{r}}$ of the dualantenna receiver $\left(L_{\mathrm{r}}=2\right)$ is given by

$$
\Sigma_{\mathrm{r}}=\left(\begin{array}{cc}
1 & 0.4 \\
0.4 & 1
\end{array}\right)
$$

The BER performance evaluation results are shown for $M$-QAM signaling, with $M=\{4,16,64,256\}$ and a $3 \times 2$ Nakagami- $m$ MIMO channel satisfying

$$
\left\{\begin{array}{l}
\Omega_{\ell, 1}=\Omega_{\ell, 2}=1 \\
\Omega_{\ell, 3}=t
\end{array}\right.
$$

with $\Omega_{\ell, k}=\mathbb{E}\left[\left|\mathbf{H}_{\ell, k}\right|^{2}\right], \ell=\{1,2\}$, and $t=\{1,0.5\}$. Note that halving the average energy transfer between the third transmit antenna and the receiver causes a BER degradation through a horizontal shift of the BER curve. In order to compare the proposed BER expressions versus direct simulations in terms of accuracy and efficiency, we assume that the ratio of the variance of the calculated or simulated BER to the square of its expectation is less or equal than a certain required accuracy $\epsilon^{2}$

$$
\frac{\operatorname{Var}\left[\hat{P}_{\mathrm{b}}\right]}{\left(\mathbb{E}\left[\hat{P}_{\mathrm{b}}\right]\right)^{2}} \leq \epsilon^{2}
$$

where $\hat{P}_{\mathrm{b}}$ denotes the BER estimate, which is given by $\hat{P}_{\mathrm{b}}=(1 / N) \sum_{l=1}^{N} X_{l}$. In case the proposed BER expressions are evaluated through Monte Carlo integration with importance sampling, $N$ and $X_{l}$ denote the number $\mathcal{N}$ of generated sample vectors $\left(\mathbf{v}_{\nu}\right)$ and the summand in (29), respectively, whereas for direct simulations, $N$ and $X_{l}$ denote the number of simulated data frames and the ratio of the number of bit errors counted in the $l$ th frame to the total number of bits within one frame, respectively. As $X_{l}$ 's are independently generated, $\operatorname{Var}\left[\hat{P}_{\mathrm{b}}\right]=(1 / N) \operatorname{Var}\left[X_{l}\right]$, where $\operatorname{Var}\left[X_{l}\right]$ can be approximated by

$$
\operatorname{Var}\left[X_{l}\right] \approx \frac{1}{N} \sum_{l=1}^{N} X_{l}^{2}-\left(\frac{1}{N} \sum_{l=1}^{N} X_{l}\right)^{2}
$$

Taking (63) into account and replacing $\mathbb{E}\left[\hat{P}_{\mathrm{b}}\right]$ in (62) by $\hat{P}_{\mathrm{b}}$, it follows that for a given accuracy $\epsilon^{2}, N$ needs to satisfy

$$
N \geq \frac{\operatorname{Var}\left[X_{l}\right]}{\epsilon^{2} \hat{P}_{\mathrm{b}}^{2}} .
$$

In Fig. 5, we have plotted the minimum $N$ for both direct simulations and the evaluation of the proposed BER expressions through Monte Carlo integration with importance sampling, for $M=4, t=1$, and a given accuracy of $\epsilon^{2}=0.01$. Note that the number of required sample vectors $\mathbf{v}_{\nu}$ in case of Monte Carlo integration with importance sampling is much less than the number of generated frames in case of direct simulations, especially for moderate to high SNR. Moreover, generating a data frame and detecting it after it has been affected by fading and channel noise, is a much more complex task than evaluating the summand in (29). In Fig. 4, the BER results from Monte Carlo integration with importance sampling have been obtained with $\mathcal{N}=5000$ samples for each RV. Note also that retaining only one term in the summation in (37) yields very accurate BER results, i.e., the BERs related to the in-phase and quadrature-phase bits of $s_{i}$ are nearly identical and irrespective of $i$. Because the number of pilot symbols is relatively high $\left(K_{\mathrm{p}}=16\right)$, the approximate BER curves resulting from the high-SNR approximation in Section $V$ also provide very accurate BER results. Only for 4-QAM, a clear deviation from the exact BER can be observed for SNRs below $10 \mathrm{~dB}$. In order not to overload Fig. 4, however, the approximate BER curves are omitted in the figure.

Fig. 6 illustrates the BER performance versus the number of pilot symbols $K_{\mathrm{p}}$ for the $3 \times 4$ OSTBC given by (59), operating over correlated i.d. Nakagami- $m$ fading channels with $m=2$ and $\Omega=1$, under the assumption that $E_{\mathrm{b}} / N_{0}=10 \mathrm{~dB}$. The power correlation matrix $\Sigma_{\mathrm{r}}$ of the dual-antenna receiver $\left(L_{\mathrm{r}}=2\right)$ is assumed to be given by (60), the channel is recovered through LS channel estimation, and the transmitted information symbols belong to a 16-QAM constellation. The results are shown for $K=\{100,400,1000\}$ information symbols and for the power correlation matrix $\Sigma_{\mathrm{t}}$ at the transmitter side being given by either (57) (lin) or the identity matrix (unc). From Fig. 6, we observe that the optimal number of pilot symbols grows with the number of information symbols $K$ and that antenna correlation does not affect this optimal number. We also notice that for large $K$, obtaining the optimal number of pilot 


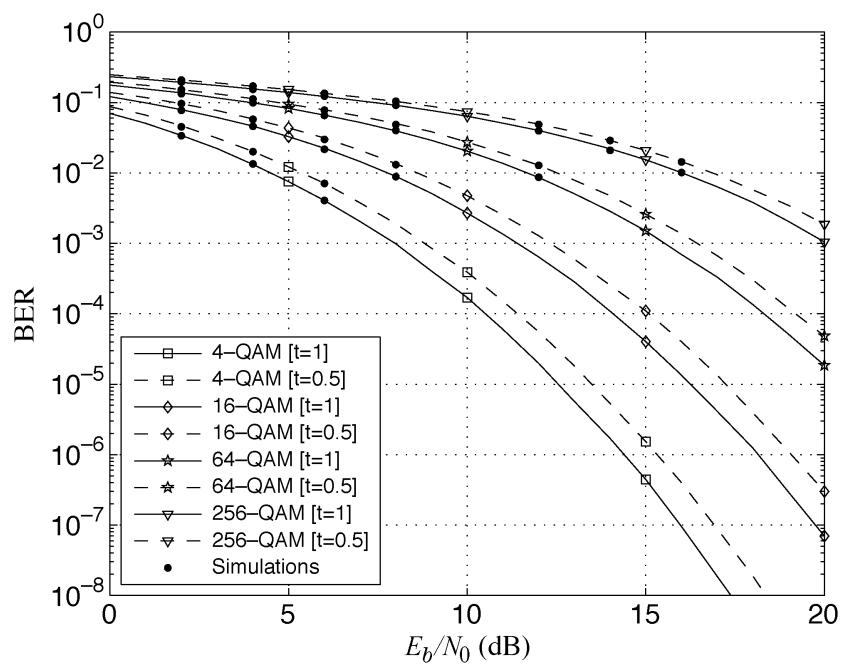

Fig. 4. BER versus $E_{\mathrm{b}} / N_{0}$ for the $3 \times 4$ OSTBC given by (59) operating over a $3 \times 2$ correlated Nakagami- $m$ MIMO fading channel with $m=2$, $\Omega_{\ell, 1}=\Omega_{\ell, 2}=1$, and $\Omega_{\ell, 3}=t$. The results are shown for $M$-QAM, with $M=\{4,16,64,256\}$, and for $t=\{1,0.5\}$.

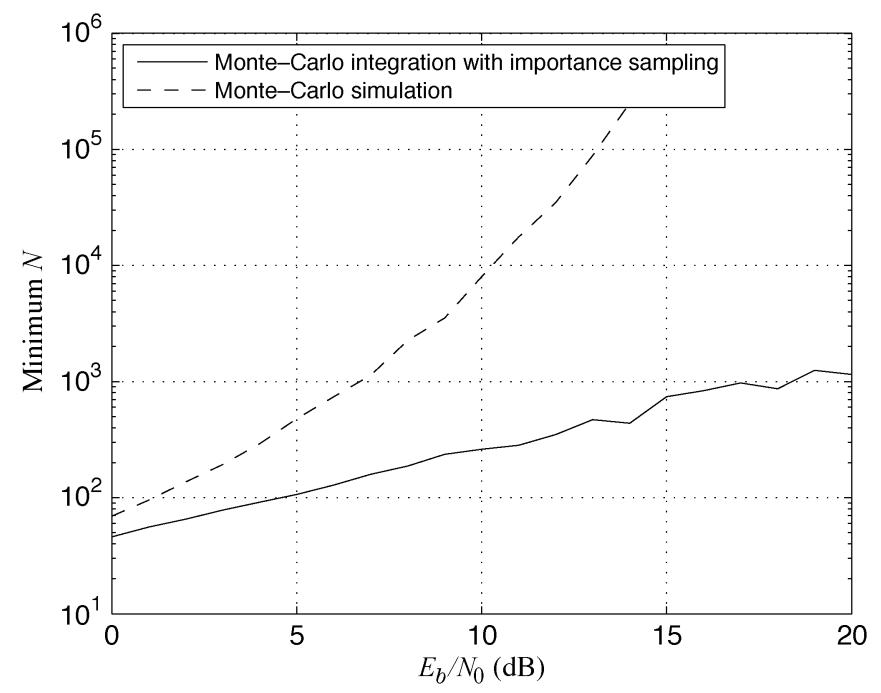

Fig. 5. Minimum $N$ for both direct simulations and the evaluation of the proposed BER expressions through Monte Carlo integration with importance sampling for a given accuracy of $\epsilon^{2}=0.01$.

symbols is not very critical as the BER grows only slowly when more pilot symbols are added.

\section{CONCLusions ANd Remarks}

In this contribution, we have investigated the effect of ICSI on the BER performance of OSTBCs under flat-fading channels. For nonsquare OSTBCs, the resulting exact BER expression can be written as an expectation over $N_{\mathrm{s}}$ discrete RVs and $2 L+3$ real-valued continuous RVs, whereas for square OSTBCs, the resulting exact BER expression reduces to an expectation over $N_{\mathrm{s}}$ discrete RVs and 4 real-valued continuous RVs, regardless of the number of antennas. The exact BER expressions can be efficiently and accurately evaluated by means of numerical integration techniques, i.e., the quadrature rule and Monte Carlo integration with importance sampling, or a combination thereof. Additionally, we provided a simple approxima-

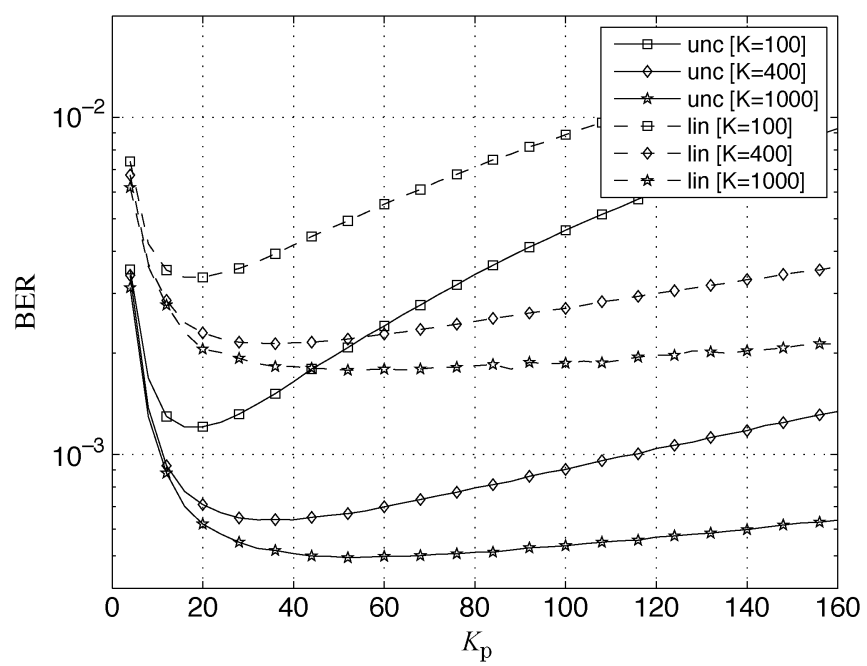

Fig. 6. BER versus $K_{\mathrm{p}}$ for the $3 \times 4$ OSTBC given by (59) with 16-QAM, operating over i.d. correlated Nakagami- $m$ fading channels, with $m=2$ and $\Omega=1$. The results are shown for $E_{\mathrm{b}} / N_{0}=10 \mathrm{~dB}$, for uncorrelated (unc) and linear (lin) correlation matrices at the transmitter side, and for $K=\{100,400,1000\}$.

tion of the BER based on treating the symbol interference due to imperfect channel estimation as white Gaussian noise. Although the resulting expression is in general not asymptotically exact, it yields very accurate BER results when the fading distribution is similar to Rayleigh and when a sufficient number $K_{\mathrm{p}}$ of pilot symbols is used. For the case of correlated Nakagami- $m$ fading channels, we elaborated further on the numerical evaluation of the exact BER expressions. Our numerical results illustrate the effect of channel estimation errors and of fading correlation on the BER performance of OSTBCs in the case of Nakagami- $m$ fading.

When an outer channel code is concatenated with an OSTBC scheme, the exact BER performance of the system is hard to assess. However, an upper bound on the BER performance can be easily derived from the PEPs, which can be obtained exactly using the techniques that are proposed in the paper.

\section{APPENDIX A}

PROOF OF (23)

In (19), we have defined $u_{i}^{\prime}$ as

$$
u_{i}^{\prime} \triangleq \frac{\operatorname{tr}\left(\mathbf{C}_{i}^{H} \hat{\mathbf{H}}^{H} \mathbf{H} \mathbf{C}+\mathbf{C}^{H} \mathbf{H}^{H} \hat{\mathbf{H}} \mathbf{C}_{i}^{\prime}\right)}{\lambda\|\hat{\mathbf{H}}\|_{\mathrm{F}}^{2}} .
$$

With $\mathrm{q}=\mathrm{R}$ and $\mathrm{q}=\mathrm{I}$ referring to the BER computation for the in-phase and quadrature-phase bits, respectively, it is readily verified that the real and imaginary parts of (65) can be rewritten as

$$
u_{i, \mathrm{q}}^{\prime}=\frac{1}{\lambda\|\hat{\mathbf{h}}\|^{2}} \sum_{n=1}^{N_{\mathrm{s}}}\left\{\left[\hat{\mathbf{h}}^{T} \mathbf{g}_{n, \mathrm{R}}^{(i, \mathrm{q})}\right] s_{n, \mathrm{R}}+\left[\hat{\mathbf{h}}^{T} \mathbf{g}_{n, \mathrm{I}}^{(i, \mathrm{q})}\right] s_{n, \mathrm{I}}\right\}
$$

where the $2 L \times 1$ column vectors $\mathbf{g}_{n, \mathrm{p}}^{(i, \mathrm{q})}$, with $\mathrm{p}=\mathrm{R}$ or $\mathrm{p}=\mathrm{I}$, are given by

$$
\mathbf{g}_{n, \mathrm{p}}^{(i, \mathrm{q})} \triangleq\left[\mathbf{M}_{n, \mathrm{p}}^{(i, \mathrm{q})} \otimes \mathbf{I}_{L_{\mathrm{r}}}\right] \mathbf{h}
$$


and the $2 L_{\mathrm{t}} \times 2 L_{\mathrm{t}}$ matrices $\mathbf{M}_{n, \mathrm{p}}^{(i, \mathrm{q})}$ are defined as

$$
\mathbf{M}_{n, \mathrm{p}}^{(i, \mathrm{q})} \triangleq\left(\begin{array}{ccc}
\mathbf{M}_{n, \mathrm{p}}^{(i, \mathrm{q})}(1,1) & \ldots & \mathbf{M}_{n, \mathrm{p}}^{(i, \mathrm{p})}\left(1, L_{\mathrm{t}}\right) \\
\vdots & \ddots & \vdots \\
\mathbf{M}_{n, \mathrm{p}}^{(i, \mathrm{q})}\left(L_{\mathrm{t}}, 1\right) & \ldots & \mathbf{M}_{n, \mathrm{p}}^{(i, \mathrm{q})}\left(L_{\mathrm{t}}, L_{\mathrm{t}}\right)
\end{array}\right) .
$$

For all possible combinations of $\mathrm{p}$ and $\mathrm{q}$, the $2 \times 2$ matrices $\mathbf{M}_{n, \mathrm{p}}^{(i, \mathrm{q})}(k, \ell)$ in (68), with $1 \leq k, \ell \leq L_{\mathrm{t}}$, are defined as follows:

$$
\mathbf{M}_{n, \mathrm{R}}^{(i, \mathrm{R})}(k, \ell) \triangleq\left(\begin{array}{cc}
\alpha_{n, \mathrm{R}}^{(i, \mathrm{R})}(k, \ell) & -\beta_{n, \mathrm{R}}^{(i, \mathrm{R})}(k, \ell) \\
\beta_{n, \mathrm{R}}^{(i, \mathrm{R})}(k, \ell) & \alpha_{n, \mathrm{R}}^{(i, \mathrm{R})}(k, \ell)
\end{array}\right)
$$

with $\alpha_{n, \mathrm{R}}^{(i, \mathrm{R})}(k, \ell)$ and $\beta_{n, \mathrm{R}}^{(i, \mathrm{R})}(k, \ell)$ being the real and imaginary parts of $\left[\left(\mathbf{C}_{n}+\mathbf{C}_{n}^{\prime}\right)\left(\mathbf{C}_{i}+\mathbf{C}_{i}^{\prime}\right)^{H}\right]_{\ell, k}$, respectively;

$$
\mathbf{M}_{n, \mathrm{I}}^{(i, \mathrm{R})}(k, \ell) \triangleq\left(\begin{array}{cc}
-\beta_{n, \mathrm{I}}^{(i, \mathrm{R})}(k, \ell) & -\alpha_{n, \mathrm{I}}^{(i, \mathrm{R})}(k, \ell) \\
\alpha_{n, \mathrm{I}}^{(i, \mathrm{R})}(k, \ell) & -\beta_{n, \mathrm{I}}^{(i, \mathrm{R})}(k, \ell)
\end{array}\right)
$$

with $\alpha_{n, \mathrm{I}}^{(i, \mathrm{R})}(k, \ell)$ and $\beta_{n, \mathrm{I}}^{(i, \mathrm{R})}(k, \ell)$ being the real and imaginary parts of $\left[\left(\mathbf{C}_{n}-\mathbf{C}_{n}^{\prime}\right)\left(\mathbf{C}_{i}+\mathbf{C}_{i}^{\prime}\right)^{H}\right]_{\ell, k}$, respectively;

$$
\mathbf{M}_{n, \mathrm{R}}^{(i, \mathrm{I})}(k, \ell) \triangleq\left(\begin{array}{cc}
\beta_{n, \mathrm{R}}^{(i, \mathrm{I})}(k, \ell) & \alpha_{n, \mathrm{R}}^{(i, \mathrm{I})}(k, \ell) \\
-\alpha_{n, \mathrm{R}}^{(i, \mathrm{I})}(k, \ell) & \beta_{n, \mathrm{R}}^{(i, \mathrm{I})}(k, \ell)
\end{array}\right)
$$

with $\alpha_{n, \mathrm{R}}^{(i, \mathrm{I})}(k, \ell)$ and $\beta_{n, \mathrm{R}}^{(i, \mathrm{I})}(k, \ell)$ being the real and imaginary parts of $\left[\left(\mathbf{C}_{n}+\mathbf{C}_{n}^{\prime}\right)\left(\mathbf{C}_{i}-\mathbf{C}_{i}^{\prime}\right)^{H}\right]_{\ell, k}$, respectively;

$$
\mathbf{M}_{n, \mathrm{I}}^{(i, \mathrm{I})}(k, \ell) \triangleq\left(\begin{array}{cc}
\alpha_{n, \mathrm{I}}^{(i, \mathrm{I})}(k, \ell) & -\beta_{n, \mathrm{I}}^{(i, \mathrm{I})}(k, \ell) \\
\beta_{n, \mathrm{I}}^{(i, \mathrm{I})}(k, \ell) & \alpha_{n, \mathrm{I}}^{(i, \mathrm{I}}(k, \ell)
\end{array}\right)
$$

with $\alpha_{n, \mathrm{I}}^{(i, \mathrm{I})}(k, \ell)$ and $\beta_{n, \mathrm{I}}^{(i, \mathrm{I})}(k, \ell)$ being the real and imaginary parts of $\left[\left(\mathbf{C}_{n}-\mathbf{C}_{n}^{\prime}\right)\left(\mathbf{C}_{i}-\mathbf{C}_{i}^{\prime}\right)^{H}\right]_{\ell, k}$, respectively.

Note that $\alpha_{i, \mathrm{q}}^{(i, \mathrm{q})}(k, \ell)=\lambda \delta_{k-\ell}$ and $\beta_{i, \mathrm{q}}^{(i, \mathrm{q})}(k, \ell)=0$, such that $\mathbf{g}_{i, \mathrm{q}}^{(i, \mathrm{q})}=\lambda \mathbf{h}$. Under the assumption that $\breve{\mathrm{q}}=\mathrm{I}$ or $\breve{\mathrm{q}}=\mathrm{R}$ when $\mathrm{q}=\mathrm{R}$ or $\mathrm{q}=\mathrm{I}$, respectively, it follows from (4) that, for any given $i$, the vectors $\mathbf{g}_{n, \mathrm{q}}^{(i, \mathrm{q})}$, with $n \neq i$, and $\mathbf{g}_{n, \breve{\mathrm{q}}}^{(i, \mathrm{q})}$ are orthogonal to $\mathrm{g}_{i, \mathrm{q}}^{(i, \mathrm{q})}$

$$
\begin{aligned}
& {\left[\mathbf{g}_{i, \mathrm{q}}^{(i, \mathrm{q})}\right]^{T} \mathbf{g}_{n, \mathrm{q}}^{(i, \mathrm{q})}=\lambda^{2}\|\mathbf{h}\|^{2} \delta_{i-n}} \\
& {\left[\mathbf{g}_{i, \mathrm{q}}^{(i, \mathrm{q})}\right]^{T} \mathbf{g}_{n, \mathrm{q}}^{(i, \mathrm{q})}=0}
\end{aligned}
$$

In (66), the term in $s_{i, \mathrm{q}}$ is the useful term, whereas the terms in $s_{n, \mathrm{q}}$, with $n \neq i$, and the terms in $s_{n, \breve{\mathrm{q}}}$ represent interference from the symbol components different from $s_{i, \mathrm{q}}$. In case of PCSI, i.e., $\hat{\mathbf{h}}=\mathbf{h}$, it is readily verified that $u_{i, \mathrm{q}}^{\prime}=s_{i, \mathrm{q}}$. Let us now introduce the $2 L \times 2 L$ matrix $\mathbf{M}_{i, \mathrm{q}}$ as

$$
\mathbf{M}_{i, \mathrm{q}} \triangleq\left\{\sum_{\substack{n=1 \\ n \neq i}}^{N_{s}} s_{n, \mathrm{q}} \mathbf{M}_{n, \mathrm{q}}^{(i, \mathrm{q})}+\sum_{n=1}^{N_{s}} s_{n, \breve{\mathrm{q}}} \mathbf{M}_{n, \mathrm{q}}^{(i, \mathrm{q})}\right\} \otimes \mathbf{I}_{L_{\mathrm{r}}}
$$

which is a function of the transmitted symbol vector $\mathbf{s}$ and the coefficient matrices $\mathbf{C}_{n}$ and $\mathbf{C}_{n}^{\prime}$, with $1 \leq n \leq N_{\mathrm{s}}$. In this way, (66) reduces to

$$
u_{i, \mathrm{q}}^{\prime}=\frac{1}{\lambda\|\hat{\mathbf{h}}\|^{2}}\left(\lambda s_{i, \mathrm{q}} \hat{\mathbf{h}}^{T} \mathbf{h}+\hat{\mathbf{h}}^{T} \mathbf{M}_{i, \mathrm{q}} \mathbf{h}\right) .
$$

From (67) and (70), it follows that $\mathbf{M}_{i, \mathrm{q}} \mathbf{h}$ is orthogonal to $\mathbf{h}$. Hence, we can define an orthonormal coordinate system with $2 L$ unit vectors $\left\{\mathbf{e}_{n}^{(i, \mathrm{q})}, n=1,2, \ldots, 2 L\right\}$, where $\mathbf{e}_{1}^{(i, \mathbf{q})}$ and $\mathbf{e}_{2}^{(i, \mathbf{q})}$ are directed along $\mathbf{h}$ and $\mathbf{M}_{i, \mathrm{q}} \mathbf{h}$, respectively. Denoting by

$$
x_{n}^{(i, \mathrm{q}) \triangleq} \hat{\mathbf{h}}^{T} \mathbf{e}_{n}^{(i, \mathrm{q})}
$$

the projections of $\hat{\mathbf{h}}$ on $\mathbf{e}_{n}^{(i, \mathrm{q})}$, it follows from (10) that the statistical properties of the RVs $x_{n}^{(i, q)}$ are independent of $i$ and q. Therefore, we may drop the superscript $(i, \mathrm{q})$, resulting in

$$
\|\hat{\mathbf{h}}\|^{2}=x_{1}^{2}+x_{2}^{2}+z^{2}
$$

with

$$
z=\sqrt{\sum_{n=3}^{2 L} x_{n}^{2}}
$$

Taking the specific choice of $\mathbf{e}_{1}^{(i, \mathrm{q})}$ and $\mathbf{e}_{2}^{(i, \mathrm{q})}$ into account, it follows from (73)-(75) that (72) reduces to (23).

\section{APPENDIX B}

PROOF OF (25)

For square OSTBCs, i.e., $L_{\mathrm{t}}=K_{\mathrm{c}}$, it follows from (5) that the vectors $\mathbf{g}_{n, \mathrm{q}}^{(i, \mathrm{q})}$ and $\mathbf{g}_{n, \breve{q}}^{(i, \mathrm{q})}$, given by (67), are mutually orthogonal, such that the orthogonality conditions given by (70) reduce to

$$
\begin{aligned}
& {\left[\mathbf{g}_{n, \mathrm{p}}^{(i, \mathrm{q})}\right]^{T} \mathbf{g}_{n^{\prime}, \mathrm{p}}^{(i, \mathrm{q})}=\lambda^{2}\|\mathbf{h}\|^{2} \delta_{n-n^{\prime}}} \\
& {\left[\mathbf{g}_{n, \mathrm{q}}^{(i, \mathrm{q})}\right]^{T} \mathbf{g}_{n^{\prime}, \mathrm{q}}^{(i, \mathrm{q})}=0 .}
\end{aligned}
$$

Using the above properties, it can be shown that $\left\|\mathbf{M}_{i, \mathrm{q}} \mathbf{h}\right\|$ which appears in (23), with $\mathbf{M}_{i, \mathrm{q}}$ given by (71), reduces to (25).

\section{REFERENCES}

[1] S. M. Alamouti, "A simple transmit diversity technique for wireless communications," IEEE J. Sel. Areas Commun., vol. 16, pp. 1459-1478, Oct. 1998.

[2] V. Tarokh, H. Jafarkhani, and A. R. Calderbank, "Space-time block codes from orthogonal designs," IEEE Trans. Inf. Theory, vol. 45, no. 5, pp. 1456-1467, Jul. 1999.

[3] X.-B. Liang, "Orthogonal designs with maximal rates," IEEE Trans. Inf. Theory, vol. 49, no. 10, pp. 2468-2503, Oct. 2003.

[4] I.-M. Kim and V. Tarokh, "Variable-rate space-time block codes in m-ary PSK systems," IEEE J. Sel. Areas Commun., vol. 21, no. 3, pp. 362-373, Apr. 2003.

[5] G. Femenias, "BER performance of linear STBC from orthogonal designs over MIMO correlated Nakagami- $m$ fading channels," IEEE Trans. Veh. Technol., vol. 53, no. 2, pp. 307-317, Mar. 2004.

[6] S.-H. Kim, I.-S. Kang, and J.-S. No, "Exact bit error probability of orthogonal space-time block codes with QAM," in Proc. IEEE Int. Symp. Inf. Theor. (ISIT), Yokohama, Japan, Jun. 29-Jul. 4, 2003, p. 63.

[7] J. Yuan, "On the performance of space-time block codes on slow Rayleigh fading channels," in Proc. IEEE Int. Symp. Personal, Indoor Mobile Radio Commun. (PIMRC), Beijing, China, Sep. 7-10, 2003, vol. 2, pp. 1688-1692.

[8] H. Zhang and T. A. Gulliver, "Capacity and error probability analysis for orthogonal space-time block codes over fading channels," IEEE Trans. Wireless Commun., vol. 4, no. 2, pp. 808-819, Mar. 2005.

[9] I.-M. Kim, "Exact BER analysis of OSTBCS in spatially correlated MIMO channels," IEEE Trans. Commun., vol. 54, no. 8, pp. 1365-1373, Aug. 2006.

[10] A. Maaref and S. Aissa, "Performance analysis of orthogonal spacetime block codes in spatially correlated MIMO Nakagami fading channels," IEEE Trans. Wireless Commun., vol. 5, no. 4, pp. 807-817, Apr. 2006. 
[11] G. Ropokis, A. Rontogiannis, and P. Mathiopoulos, "Quadratic forms in normal rvs: Theory and applications to OSTBC over Hoyt fading channels," IEEE Trans. Wireless Commun., vol. 7, no. 12, pp. 5009-5019, Dec. 2008.

[12] J. K. Cavers, "An analysis of pilot symbol assisted modulation for Rayleigh fading channels," IEEE Trans. Veh. Technol., vol. 40, no. 4, pp. 686-693, Nov. 1991.

[13] A. L. Swindlehurst and G. Leus, "Blind and semi-blind equalization for generalized space-time block codes," IEEE Trans. Signal Process., vol. 50, no. 10, pp. 2489-2498, Oct. 2002.

[14] N. Ammar and Z. Ding, "Blind channel identifiability for generic linear space-time block codes," IEEE Trans. Signal Process., vol. 55, no. 1, pp. 202-217, Jan. 2007.

[15] L. Jacobs and M. Moeneclaey, "Effect of MMSE channel estimation on BER performance of orthogonal space-time block codes in Rayleigh fading channels," IEEE Trans. Commun., vol. 57, no. 5, pp. 1242-1245, May 2009.

[16] L. Jacobs and M. Moeneclaey, "BER analysis of square OSTBCS with LMMSE channel estimation in arbitrarily correlated Rayleigh fading channels," IEEE Commun. Lett., vol. 14, no. 7, pp. 626-628, Jul. 2010.

[17] C. Shan, P. Y. Kam, and A. Nallanathan, "A symbol-by-symbol channel estimation receiver for space-time block coded systems and its performance analysis on the nonselective Rayleigh fading channel," IEEE Trans. Commun., vol. 56, no. 12, pp. 2116-2124, Dec. 2008.

[18] M. Brehler and M. K. Varanasi, "Asymptotic error probability analysis of quadratic receivers in Rayleigh-fading channels with applications to a unified analysis of coherent and noncoherent space-time receivers," IEEE Trans. Inf. Theory, vol. 47, no. 6, pp. 2383-2399, Sep. 2001.

[19] P. Garg, R. K. Mallik, and H. M. Gupta, "Performance analysis of space-time coding with imperfect channel estimation," IEEE Trans. Wireless Commun., vol. 4, no. 1, pp. 257-265, Jan. 2005.

[20] R. K. Mallik and P. Garg, "Performance of optimum and suboptimum receivers for space-time coded systems in correlated fading," IEEE Trans. Commun., vol. 57, no. 5, pp. 1237-1241, May 2009.

[21] P. Garg, R. K. Mallik, and H. M. Gupta, "Exact error performance of square orthogonal space-time block coding with channel estimation," IEEE Trans. Commun., vol. 54, no. 3, pp. 430-437, Mar. 2006.

[22] L. Jacobs and M. Moeneclaey, "Comments on "exact error performance of square orthogonal space-time block coding with channel estimation"," IEEE Trans. Commun., vol. 57, no. 11, pp. 3249-3251, Nov. 2009.

[23] H. Cheon and D. Hong, "Performance analysis of space-time block codes in time-varying Rayleigh fading channels," in Proc. IEEE Int. Conf. Acoustics, Speech, Signal Process. (IEEE ICASSP), Orlando, FL, May 13-17, 2002, vol. 3, pp. 2357-2360.

[24] G. Ganesan and P. Stoica, "Space-time block codes: A maximum SNR approach," IEEE Trans. Inf. Theory, vol. 47, no. 4, pp. 1650-1656, May 2001.

[25] G. Taricco and E. Biglieri, "Space-time decoding with imperfect channel estimation," IEEE Trans. Wireless Commun., vol. 4, no. 4, pp. 1874-1888, Jul. 2005.

[26] M. K. Simon and M.-S. Alouini, Digital Communication Over Fading Channels, 2nd ed. New York: Wiley, 2005.

[27] M. Evans, N. Hastings, and B. Peacock, Statistical Distributions 3rd Ed. New York: Wiley, 2000.

[28] W. H. Press, S. A. Teukolsky, W. T. Vetterling, and B. P. Flannery, Numerical Recipes: The Art of Scientific Computing, 3rd ed. New York: Cambridge Univ. Press, 2007.

[29] M. Nakagami, "The m-distribution-A general formula for intensity distribution of rapid fading," in Statistical Methods in Radio Wave Propagation. New York: Pergamon, 1960, pp. 3-36.

[30] I. S. Gradshteyn and I. M. Ryzhik, Table of Integrals, Series, and Products, 6th ed. New York: Academic, 2000.

[31] Z. Chen, Z. Chi, and B. Vucetic, "Error performance of maximal-ratio combining with transmit antenna selection in Nakagami- $m$ fading channels," in Proc. Int. Conf. Wireless Communications, Networking, Mobile Comput. (WiCOM), Wuhan, China, Sep. 22-24, 2006, pp. 1-3.

[32] K. I. Pedersen, J. B. Andersen, J. P. Kermoal, and P. Mogensen, "A stochastic multiple-input-multiple-output radio channel model for evaluation of space-time coding algorithms," in Proc. IEEE Vehicular Technol. Conf. (IEEE VTC Fall), Boston, MA, Sep. 24-28, 2000, vol. 2, pp. 893-897.

[33] M.-S. Alouini, A. Abdi, and M. Kaveh, "Sum of gamma variates and performance of wireless communication systems over Nakagami-fading channels," IEEE Trans. Veh. Technol., vol. 50, no. 6, pp. 1471-1480, Nov. 2001.
[34] G. K. Karagiannidis, N. C. Sagias, and T. A. Tsiftsis, "Closed-form statistics for the sum of squared Nakagami- $m$ variates and its applications," IEEE Trans. Commun., vol. 54, no. 8, pp. 1353-1359, Aug. 2006.

[35] J. Reig, "Performance of maximal ratio combiners over correlated Nakagami- $m$ fading channels with arbitrary fading parameters," IEEE Trans. Wireless Commun., vol. 7, no. 5, pp. 1441-1444, May 2008.

[36] P. Lombardo, G. Fedele, and M. M. Rao, "MRC performance for binary signals in Nakagami fading with general branch correlation," IEEE Trans. Commun., vol. 47, no. 1, pp. 44-52, Jan. 1991.

[37] Q. T. Zhang, "Maximal-ratio combining over Nakagami fading channels with an arbitrary branch covariance matrix," IEEE Trans. Veh. Technol., vol. 48, no. 4, pp. 1141-1150, Jul. 1999.

[38] M. Z. Win, G. Chrisikos, and J. H. Winters, "MRC performance for $m$-ary modulation in arbitrarily correlated Nakagami fading channels," IEEE Commun. Lett., vol. 4, no. 10, pp. 301-303, Oct. 2000.

[39] G. C. Alexandropoulos, N. C. Sagias, F. I. Lazarakis, and K. Berberidis, "New results for the multivariate Nakagami- $m$ fading model with arbitrary correlation matrix and applications," IEEE Trans. Wireless Commun., vol. 8, no. 1, pp. 245-255, Jan. 2009.

[40] N. C. Beaulieu and C. Cheng, "Efficient Nakagami- $m$ fading channel simulation," IEEE Trans. Veh. Technol., vol. 54, no. 2, pp. 413-424, Mar. 2005.

[41] C. Lingzhi and N. C. Beaulieu, "Simple efficient methods for generating independent and bivariate Nakagami- $m$ fading envelope samples," IEEE Trans. Veh. Technol., vol. 56, no. 4, pp. 1573-1579, Jul. 2007.

[42] C. Meyer, Matrix Analysis and Applied Linear Algebra. Philadelphia, PA: SIAM, 2000.

[43] T. Tran and A. Sesay, "Sum of arbitrarily correlated gamma variates and performance of wireless communication systems over Nakagami- $m$ fading channels," IET Commun., vol. 1, no. 6, pp. 1133-1137, Dec. 2007.

[44] A. A. Abu-Dayya and N. C. Beaulieu, "Outage probabilities of cellular mobile radio systems with multiple Nakagami interferers," IEEE Trans. Veh. Technol., vol. 40, no. 4, pp. 757-768, Nov. 1991.

[45] C. Oestges, "Validity of the kronecker model for MIMO correlated channels," in Proc. IEEE Vehicular Technol. Conf. (IEEE VTC_Spring), Melbourne, Australia, May 7-10, 2006, vol. 6, pp. $2818-2822$.

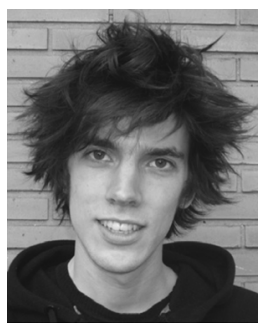

Lennert Jacobs (S'07) was born in Ghent, Belgium, in 1983. He received the Master's degree in electrical engineering from Ghent University, Gent, Belgium, in 2006. He is currently working toward the Ph.D. degree in the Department of Telecommunications and Information Processing at Ghent University.

His main research interests are in error analysis, channel estimation, MIMO techniques, and modulation and coding for wireless digital communications.

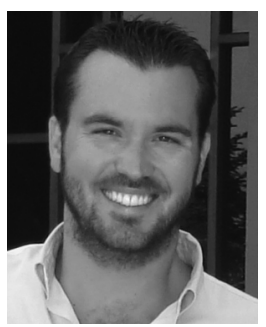

George C. Alexandropoulos (S'07-M'10) was born in Athens, Greece, in 1980. He received the Diploma degree in computer engineering and informatics, the M.A.Sc. degree (with distinction) in signal processing and communications, and the $\mathrm{Ph} . \mathrm{D}$. degree in wireless communications from the Computer Engineering and Informatics Department (CEID), School of Engineering (SE), University of Patras (UoP), Rio-Patras, Greece, in 2003, 2005, and 2010, respectively.

From 2001, he has been a Research Fellow at the Signal Processing and Communications Laboratory, CEID, SE, UoP, Rio-Patras, Greece. From 2006 to 2010, he was with the National Centre for Scientific Research-"Demokritos," Athens, Greece, where he was a Ph.D. scholar at the Wireless Communications Laboratory of the Institute of Informatics and Telecommunications. Since 2007, he has been affiliated with the National Observatory of Athens, Institute for Space Applications and Remote Sensing, Athens, Greece, where he has been participating in several national and european $R \& D$ projects. His research interests include cooperative and cognitive radio systems, fading channels, MIMO techniques, and signal processing for communications. 
Dr. Alexandropoulos acts as a reviewer for several international journals (including the IEEE TRANSACTIONS ON COMMUNICATIONS, the IEEE TRANSACTIONS ON WIRELESS COMMUNICATIONS, and the IEEE TRANSACTIONS ON Vehicular TeChNOLOGY) and IEEE conferences (ICC, GLOBECOM, VTC, etc.). He is a member of the IEEE Communications Society and the Technical Chamber of Greece. He has received a postgraduate scholarship from the Operational Programme for Education and Initial Vocational Training II, Ministry of Education, Lifelong Learning, and Religious Affairs, Greek Republic and has been awarded with a student travel grant for the IEEE GLOBECOM 2010 in Miami, Florida.

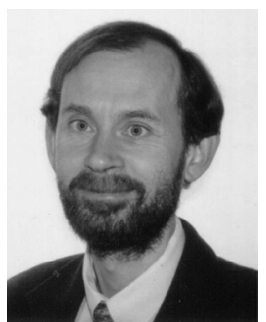

Marc Moeneclaey (M'93-SM'99-F'02) received the Electrical Engineering diploma and the Ph.D. degree in electrical engineering from Ghent University, Gent, Belgium, in 1978 and 1983, respectively.

$\mathrm{He}$ is Professor at the Department of Telecommunications and Information Processing (TELIN), Gent University. His main research interests are in statistical communication theory, carrier and symbol synchronization, bandwidth-efficient modulation and coding, spread-spectrum, satellite and mobile communication. He is the author of more than 400 scientific papers in international journals and conference proceedings. Together with Prof. H. Meyr (RWTH Aachen) and Dr. S. Fechtel (Siemens AG), he coauthored the book Digital Communication Receivers-Synchronization, Channel Estimation, and Signal Processing (Wiley, 1998).

Dr. Moeneclaey is corecipient of the Mannesmann Innovations Prize 2000. During the period 1992-1994, he was Editor for Synchronization, for the IEEE TRANSACTIONS ON COMMUNICATIONS. He served as Co-Guest Editor for special issues of the Wireless Personal Communications Journal (on Equalization and Synchronization in Wireless Communications) and the IEEE JOURNAL ON SELECTED AREAS IN COMMUNICATIONS (on Signal Synchronization in Digital Transmission Systems) in 1998 and 2001, respectively.

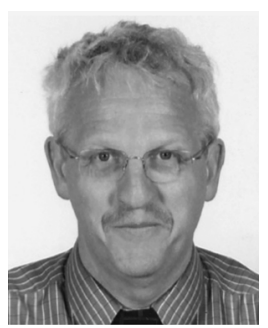

Herwig Bruneel was born in Zottegem, Belgium, in 1954. He received the Master's degree in electrical engineering, the Master's degree in computer science, , and the Ph.D. degree in computer science, all from Ghent University, Belgium, in 1978, 1979, and 1984, respectively.

$\mathrm{He}$ is full Professor in the Faculty of Engineering and head of the Department of Telecommunications and Information Processing at the same university. He also leads the SMACS Research Group within this department. His main personal research interests include stochastic modelling and analysis of communication systems, discretetime queueing theory, and the study of ARQ protocols. He has published more than 400 papers on these subjects and is coauthor, with H. Bruneel and B. G. Kim, of the book Discrete-Time Models for Communication Systems Including
ATM (Boston, MA: Kluwer, 1993). From October 2001 to September 2003, he served as the Academic Director for Research Affairs at Ghent University.

Dr. Bruneel has held a career-long Methusalem grant from the Flemish Government at Ghent University, specifically on Stochastic Modelling and Analysis of Communication Systems since 2009.

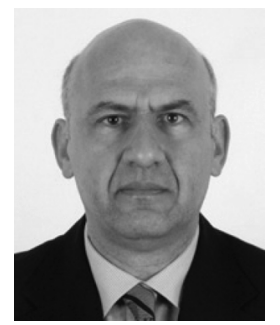

P. Takis Mathiopoulos (S'80-M'81-SM'94) received the Ph.D. degree in digital communications from the University of Ottawa, Canada, in 1989.

From 1982 to 1986, he was with Raytheon Canada, Ltd., working in the areas of air navigational and satellite communications. In 1988, he joined the Department of Electrical and Computer Engineering (ECE), University of British Columbia (UBC), Canada, where he was a faculty member for 13 years, last holding the rank of Professor. He is currently Director of Research at the Institute for Space Applications and Remote Sensing (ISARS), National Observatory of Athens (NOA), where he established the Wireless Communications Research Group. As ISARS' Director (2000-2004), he has led the Institute to a significant expansion, R\&D growth, and international scientific recognition. For these achievements, ISARS has been selected as a national Centre of Excellence for the years 2005 to 2008. Since 2003, he has also taught part-time at the Department of Informatics and Telecommunications, University of Athens, where he was recently elected Professor of Digital Communications. In 2008 and for a period of five years, he was appointed Guest Professor at the Southwest Jiaotong University, China. For the last 20 years, he has been conducting research mainly on the physical layer of digital communication systems for terrestrial and satellite applications, including digital communications over fading and interference environments. He coauthored a paper in GLOBECOM' 89 establishing for the first time in the open technical literature the link between MLSE and multiple (or multi-symbol) differential detection for the AWGN and fading channels. He is also interested in channel characterization and measurements, modulation and coding techniques, SIMO/MIMO, UWB, OFDM, and software/cognitive radios. In these areas, he has coauthored approximately 80 IEEE journal papers, four book chapters, and more than 110 conference papers. He has been Principal Investigator for more than 40 research grants and has supervised the thesis of 11 Ph.D. and 23 Master's students.

Dr. Mathiopoulos has been or currently serves on the editorial board of several scientific journals, including the IET Communications, and the IEEE TRANSACTIONS ON COMMUNICATIONS from 1993 to 2005. He has regularly acted as a Consultant for various governmental and private organizations. Since 1993, he has served on a regular basis as a scientific advisor and a technical expert for the European Commission (EC). He has been a member of the TPC of more than 50 international conferences, as well as the Vice Chair for the 2006-S IEEE Vehicular Technology Conference (VTC) and the 2008-F IEEE VTC. He has delivered numerous invited presentations, including plenary lectures, and has taught many short courses all over the world. He was an ASI Fellow, a Killam Research Fellow, and a corecipient of the Best Paper Award from the 2008 International Conference on Communication, Control, and Signal Processing. 